ARTICLE

httpst//doi.org/10.1038/s414.67-020-16497-x

\title{
A hypomorphic cystathionine $\beta$-synthase gene contributes to cavefish eye loss by disrupting optic vasculature
}

Li Ma (1) ${ }^{1}$, Aniket V. Gore ${ }^{2}$, Daniel Castranova ${ }^{2}$, Janet Shi ${ }^{1}$, Mandy Ng${ }^{1}$, Kelly A. Tomins ${ }^{2,3}$, Corine M. van der Weele ${ }^{1}$, Brant M. Weinstein ${ }^{2} \&$ William R. Jeffery (iD ${ }^{1 \times}$

Vestigial structures are key indicators of evolutionary descent, but the mechanisms underlying their development are poorly understood. This study examines vestigial eye formation in the teleost Astyanax mexicanus, which consists of a sighted surface-dwelling morph and multiple populations of blind cave morphs. Cavefish embryos initially develop eyes, but they subsequently degenerate and become vestigial structures embedded in the head. The mutated genes involved in cavefish vestigial eye formation have not been characterized. Here we identify cystathionine $\beta$-synthase $a(c b s a)$, which encodes the key enzyme of the transsulfuration pathway, as one of the mutated genes responsible for eye degeneration in multiple cavefish populations. The inactivation of cbsa affects eye development by increasing the transsulfuration intermediate homocysteine and inducing defects in optic vasculature, which result in aneurysms and eye hemorrhages. Our findings suggest that localized modifications in the circulatory system may have contributed to the evolution of vestigial eyes in cavefish.

\footnotetext{
${ }^{1}$ Department of Biology, University of Maryland, College Park, MD, USA. ${ }^{2}$ Division of Developmental Biology, Eunice Kennedy Shriver National Institute of Child Health and Human Development, NIH, Bethesda, MD, USA. ${ }^{3}$ Department of Genetics, Stanford University, School of Medicine, Stanford, CA, USA.

凶email: Jeffery@umd.edu
} 
$\mathrm{V}$ estigial structures, such as the reduced wings of flightless birds, the hind limb remnants of pythons, and the degenerate eyes of blind cavefish (CF), were key factors in Darwin's recognition of descent with modification during evolution. However, the genetic and physiological mechanisms responsible for the development of rudimentary structures are still poorly understood.

In the teleost Astyanax mexicanus, blind CF has been derived repeatedly from sighted surface fish (SF) ancestors ${ }^{1-3}$. The loss of eyes in A. mexicanus $\mathrm{CF}$ involves initial optic development followed by subsequent degeneration ${ }^{1,2}$. Eye primordia with a lens and retina are formed in CF embryos, but the lens undergoes massive apoptosis, the retina becomes apoptotic and disorganized, and eye growth is eventually arrested during larval development ${ }^{3-5}$. Accordingly, the rate of optic growth fails to increase, and the small non-functional eyes are overgrown by skin and connective tissue as CF larvae develop into adults. About 30 distinct CF populations have evolved in Mexican caves, and several of these CF populations have evolved vestigial eye phenotypes independently ${ }^{6,7}$. Similar eye reduction or loss occurs in many cave-dwelling species ${ }^{8}$ and animals adapted to other dark habitats ${ }^{9}$.

The existence of interfertile morphs in A. mexicanus has allowed eye degeneration to be studied by genetic methods ${ }^{2}$. These studies have shown that eye loss in the Pachón CF (PA-CF) population is controlled by multiple genetic factors ${ }^{10-12}$. Furthermore, genetic complementation shows that some of the factors involved in eye loss are the same and others are unique in different CF populations ${ }^{13}$. In addition to genetic changes, epigenetic events may also have contributed to the evolution of eye loss in $\mathrm{CF}^{14}$. Quantitative trait locus (QTL) analysis revealed about 15 non-overlapping genomic regions that are responsible for lens and eye reduction in PA-CF ${ }^{10-12,15}$. The alignment of eye QTL with the sequenced PA-CF genome has suggested many candidates for genes controlling vestigial eye formation ${ }^{15}$. However, the identities of the genes and mutations within these QTL intervals have not been established.

Here we identify cystathionine $\beta$-synthase a (cbsa), which encodes the key enzyme in the transsulfuration pathway, as one of the inactivated genes responsible for eye degeneration in multiple A. mexicanus CF populations. Studies of the hypomorphic $c b s a$ phenotype revealed a mechanism for arresting eye growth based on the accumulation of the transsulfuration intermediate homocysteine (hCys) and disruption of optic vasculature. Our findings suggest that interference with circulatory system functions by the hypomorphic $c b s a$ gene may have a crucial role in the evolution of $\mathrm{CF}$ eye degeneration.

\section{Results}

The cbsa gene as a candidate for CF eye loss. To identify a mutated gene responsible for $\mathrm{CF}$ eye loss, we examined A. mexicanus Ensembl Scaffold KB871589.1, which contains the peak marker of an eye size QTL located between the $h s f 2 b p$ and ankrd10a genes (Fig. 1a) ${ }^{11-13,15}$. According to the Ensembl AstMex 1.0.2 genome assembly, this scaffold contains 21 predicted protein-coding genes. We surveyed these genes for expression differences in SF and PA-CF by qualitative reverse transcriptase polymerase chain reaction (RT-PCR) at 40 hours postfertilization (hpf) (Fig. 1a; Supplementary Table 5), when changes associated with eye degeneration first appear in $\mathrm{CF}^{1-5}$. Since $\mathrm{CF}$ eye degeneration is a recessive trait ${ }^{2-5}$, we focused on the genes downregulated in PA-CF relative to SF and identified cars $2, \alpha A$-crystallin (cryaa), $h s f 2 b p$, and cystathionine $\beta$-synthase a (cbsa) (Fig. 1a). The cars2 gene, which encodes a mitochondrial aminoacyl-tRNA synthetase ${ }^{16}$ and is likely to function and be expressed ubiquitously, and cryaa, which was previously shown to be under trans-acting regulation during CF development ${ }^{17}$, were excluded from further study. Of the two remaining genes, the $h s f 2 b p$ gene, which encodes a heat shock transcription factor 2 binding protein ${ }^{18}$, is located in close proximity to the peak QTL marker. We used in situ hybridization to determine the pattern of $h s f 2 b p$ expression during normal culture at $25^{\circ} \mathrm{C}$ and following a 1 -h $37^{\circ} \mathrm{C}$ heat shock (Fig. 1b). The results indicated that $h s f 2 b p$ expression was ubiquitous in SF and PA-CF development, and although expression was increased by the heat shock, the increase was not particularly strong in the eyes (Fig. 1b). Thus our attention was focused on $c b s a$, which is expressed in the developing eyes in A. mexicanus and was predicted as a candidate for congenital anomaly in CF eye loss by Ingenuity Pathway Analysis of gene expression data ${ }^{15}$.

There are two $c b s$ paralogs in Astyanax: $c b s a$ and $c b s b^{15}$. We examined the expression of the paralogous $c b s$ genes during SF and PA-CF development by in situ hybridization with genespecific probes (Fig. 2a-q). At early stages of embryonic development, the patterns of $c b s a$ and $c b s b$ expression were similar in SF and PA-CF, including in the developing eye primordia. However, $c b s a$ expression was weaker in PA-CF than in SF (Fig. 2a-f), and $c b s b$ expression was generally stronger than $c b s a$ in PA-CF (Fig. 2a-f, i-n). By $40 \mathrm{~h}$ of development, $c b s a$ and $c b s b$ were expressed in many developing organs (Fig. 2g, h, o, p). The expression of $c b s a$ was strong in the SF head, including the eyes and brain, and in the heart, pectoral fin buds, liver, and myotomes (Fig. 2g), whereas in PA-CF cbsa expression was downregulated in all of these organs except the liver (Fig. 2h). Sections of SF larval heads at $40 \mathrm{hpf}$ showed cbsa expression in the lens, the ciliary marginal zone of the retina, and the optic tectum, whereas no expression was apparent in these regions in CF (Fig. 2q). The expression of $c b s b$ was similar in most of these locations in SF and PA-CF at $40 \mathrm{hpf}$, with the notable exception of the head and myotomes, where $c b s b$ expression was reduced or undetectable in both PA-CF and SF (Fig. 2o, p). The pattern of $c b s a$ and $c b s b$ expression in Astyanax SF resembled that reported in zebrafish ${ }^{19}$. The results suggest that $c b s a$ and $c b s b$ show mostly overlapping expression during SF and PA-CF development, except for the PA-CF eyes, brain, and myotomes in which neither of the $c b s$ paralogs was strongly expressed. Thus $c b s b$ expression could compensate for downregulated $c b s a$ expression in CF larvae everywhere except for the eyes, brain, and myotomes. The in situ hybridization results were confirmed by comparing $c b s a$ and $c b s b$ expression in isolated larval heads and trunks by qualitative and quantitative RT-PCR, which showed that $c b s a$, but not $c b s b$, expression was downregulated in PA-CF larval heads at $40 \mathrm{hpf}$ (Fig. 2s). The finding of no changes in cbsa levels between CF and SF trunks in qualitative RT-PCR experiments, despite strong downregulation in the myotomes by in situ hybridization, can be explained by compensating upregulation in the liver (Fig. $2 \mathrm{~h}$ ).

Eye loss is thought to have evolved independently at least twice in different A. mexicanus CF populations ${ }^{1,2,13}$. Therefore, we conducted in situ hybridization to determine whether there was also a change in cbsa expression in other CF populations and found that Tinaja CF (TI-CF), Los Sabinos CF (LS-CF), and Molino CF (MO-CF) had patterns of $c b s a$ downregulation similar to PA-CF, including strong downregulation in the eyes (Fig. 2r). These results establish $c b s a$ as a candidate for controlling eye loss in multiple CF populations.

CF $\boldsymbol{c} b s a$ gene contains hypomorphic mutations. We next conducted F1 hybrid tests to determine whether downregulation of the cbsa gene is caused by cis- or trans-acting regulation in $\mathrm{PA}-\mathrm{CF}^{15,20}$. In these experiments, SF females were crossed with 


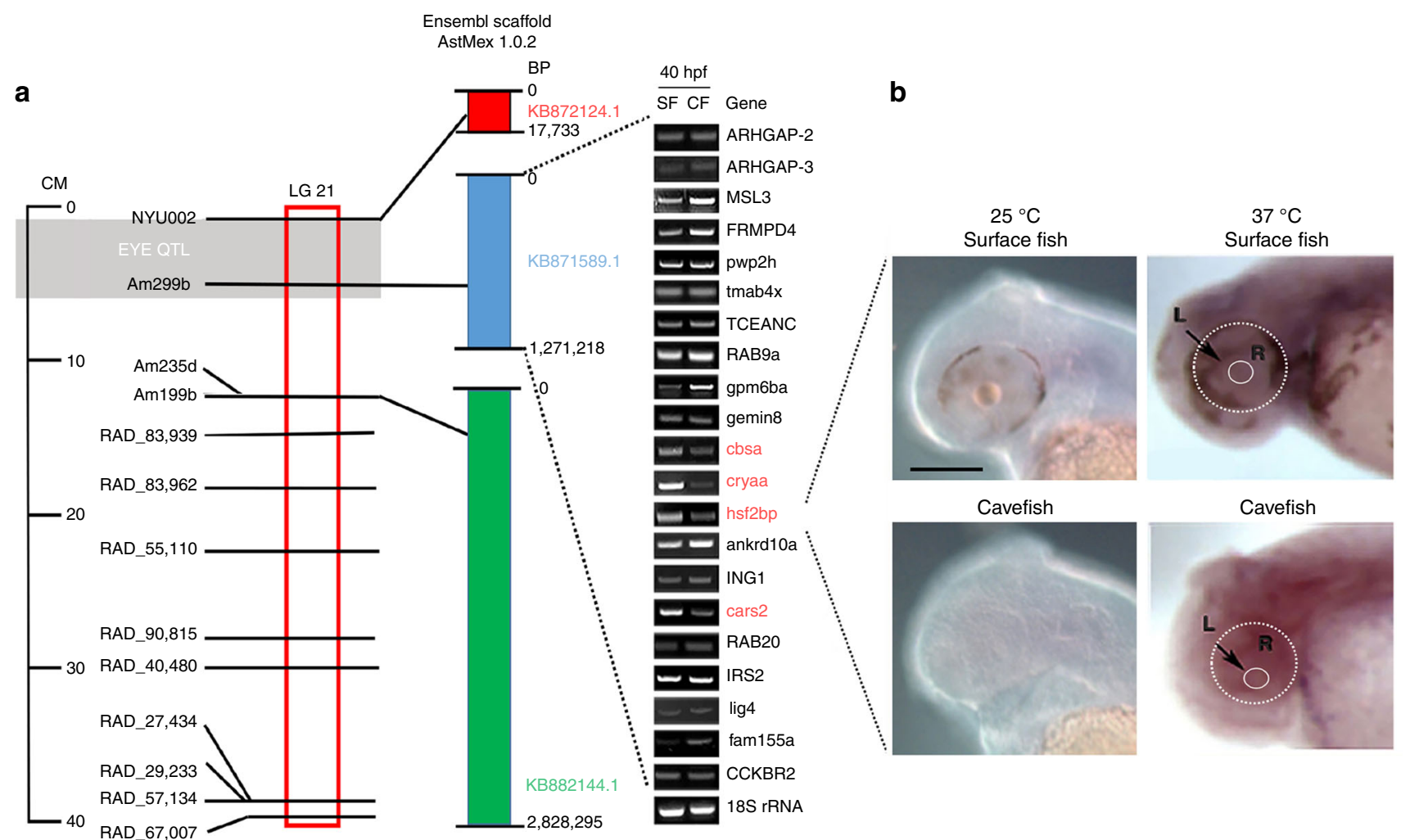

Fig. 1 Gene expression screen of PA-CF genomic scaffold KB871589.1. a Location of an eye QTL on A. mexicanus linkage group 21 (left), alignment to nearby scaffolds of the draft PA-CF (AstMex1.0.2) genome sequence based on the position of markers (center), and qualitative RT-PCR analysis of the expression of 21 predicted scaffold genes at 40 hpf (right). Expression of genes in SF and PA-CF are shown from top to bottom according to their $5^{\prime}$ to $3^{\prime}$ order on the scaffold. CM centimorgans, LG linkage group. See Supplementary Table 5 for PA-CF gene IDs. Genes downregulated in PA-CF compared to SF are shown in red. Information based on at least three biological replicates. $\mathbf{b}$ In situ hybridization showing hsf2bp expression in 40 hpf SF and PA-CF embryos at $25^{\circ} \mathrm{C}$ (left) and after treatment at $37^{\circ} \mathrm{C}$ for $1 \mathrm{~h}$ (right). Dashed lines encircle the eyes and lenses in the right frames. $n=20$ for all frames. L lens, R retina. Scale bar: $200 \mu \mathrm{m}$; same magnification in all frames. Source data are provided in source data file.

PA-CF males to generate F1 hybrids, RNA was extracted from the heads of the F1 hybrid and parental larvae at $40 \mathrm{hpf}$, and a part of the cbsa-coding region containing a single-nucleotide polymorphism marker, which distinguished the SF and CF cbsa alleles, was amplified by PCR, cloned, and sequenced (Fig. 3a). The results showed that expression of the PA-CF $c b s a$ allele was not increased in the F1 hybrid background, as expected under trans-acting control, thus implicating cis-regulation as the cause of $c b s a$ downregulation.

To identify potential cis-regulating mutations, we sequenced the SF and PA-CF cbsa loci and immediate flanking regions. The results showed that the SF and CF $c b s a$ genes consist of 15 exons (Fig. 3b). We used rapid amplification of cDNA ends (RACE) to identify the SF and CF cbsa transcripts and surveyed them for possible sequence differences. The 5 ' non-coding regions of CF and SF cbsa mRNAs, which correspond to the first non-coding exon and the first few bases of the second exon, showed only two nucleotide differences. In addition, the CF $c b s a$ coding region has no internal stop codons, suggesting that it encodes a complete cystathionine $B$-synthase A (CBSA) protein (Supplementary Fig. 1a). The deduced PA-CF and SF CBSA proteins are 99\% identical, differing by only five amino acids (Supplementary Fig. 1b). Only one of the divergent amino acids (G 257) is conserved in other known vertebrate CBS proteins. Although it is possible that PA-CF CBSA is functionally different from SF CBSA because it is missing a run of two amino acids (G257-K258; Supplementary Fig. 1c) that are present in other teleost CBS proteins, this difference would not explain the hypomorphic activity of the PA-CF $c b s a$ gene.
Outside the protein-coding regions, the $7 \mathrm{~kb}$ non-coding region located between $c b s a$ and gemin 8 , which shows $99 \%$ nucleotide identity between PA-CF and SF, has only a few substitutions, while the $5 \mathrm{~kb}$ non-coding region located between $c b s a$ and cryaa is also highly conserved in $\mathrm{SF}$ and $\mathrm{PA}-\mathrm{CF}^{17}$. In addition, $c b s a$ introns are also highly conserved in PA-CF, except for the following: a run of 4 indels in a 500-bp region of intron 1, a 323bp deletion in intron 6 , and a 120-bp insertion in intron 8 (Supplementary Table 1; Supplementary Fig. 2). To explore the possibility that these introns contain potential cis-regulatory changes, we amplified and compared their sequences in the PACF, TI-CF, LS-CF, MO-CF, Chica CF (CH-CF), and Jineo CF (JICF) $c b s a$ genes. Genetic studies have shown that eye loss evolved independently at least twice in these CF populations ${ }^{2,11,13}$, and our in situ hybridization data indicated that cbsa is downregulated in PA-CF, TI-CF, LS-CF, and MO-CF eyes (Fig. 2). We found that the indels in introns 6 and 8 were present in some, but not all, of these CF populations (Supplementary Table 1), suggesting that they are unlikely to include changes generally responsible for $c b s a$ downregulation. In contrast, indels were found at the same position in intron 1 of all six CF populations (Supplementary Fig. 2), suggesting that this region is a candidate for containing cis-acting mutations.

We next searched for enhancers in the genomic region between the 3 ' termini of the gemin 8 and the $c b s a$ genes using iEnhancer$2 \mathrm{~L}^{21}$. A putative 282-bp enhancer (E region, Fig. $3 \mathrm{~b}$ ) was predicted in $c b s a$ intron 1, which overlapped the indels described above in all six CF populations. To test for regulatory function, we made DNA constructs in which a 354-bp genomic DNA 


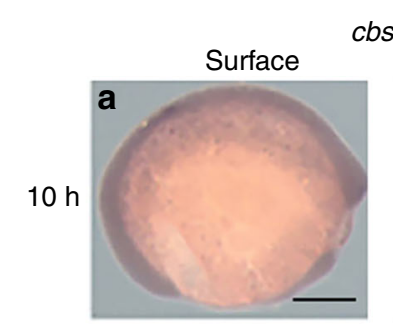

cbsa
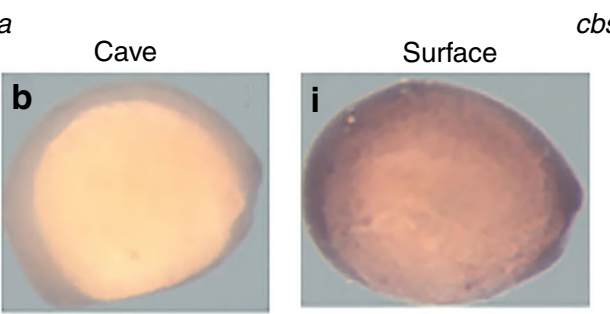

$c b s b$
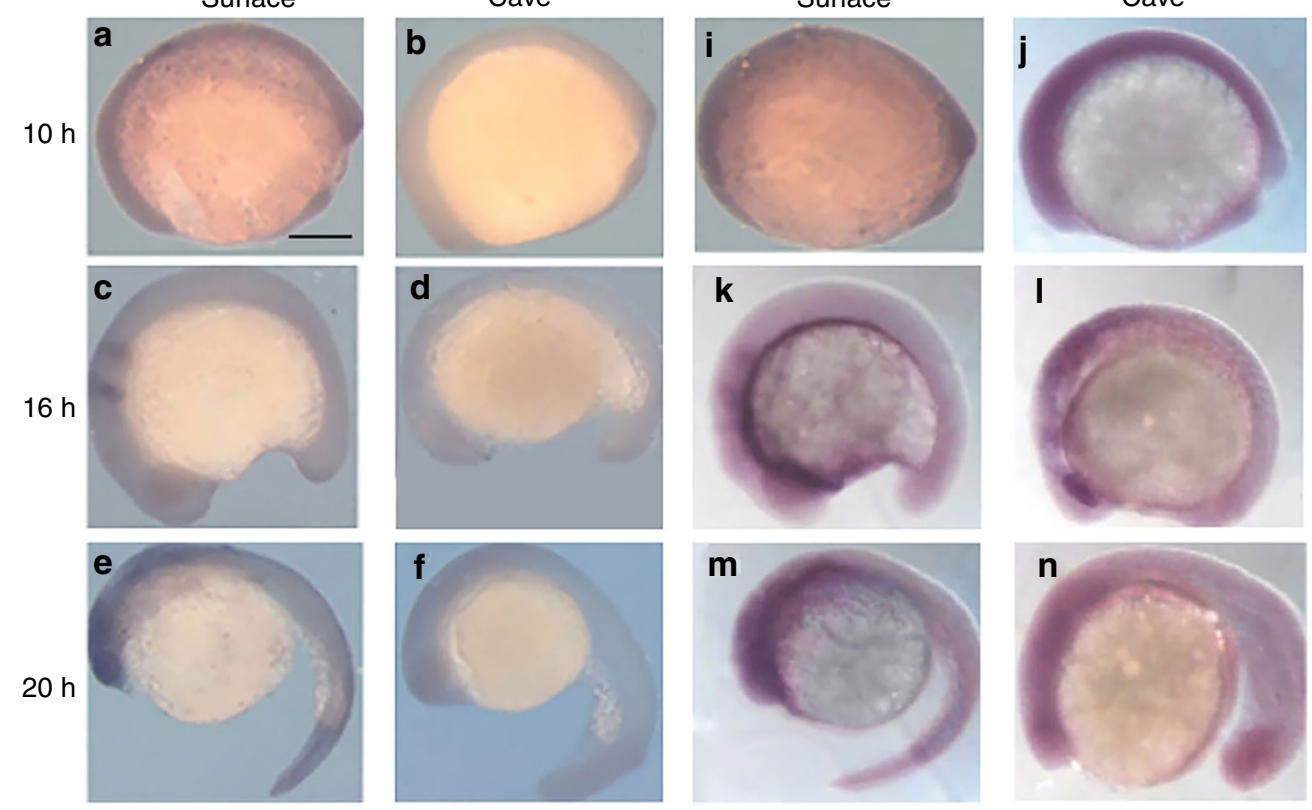

Surface

9

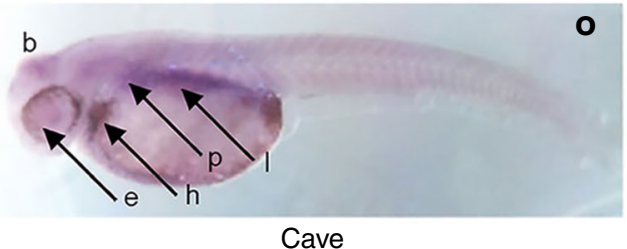

$40 \mathrm{~h}$
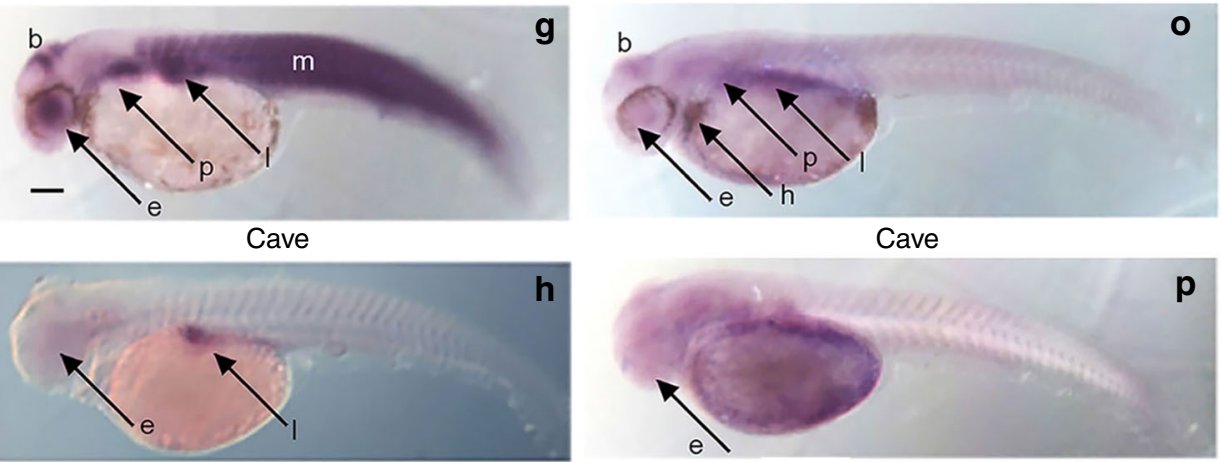

$40 \mathrm{~h}$

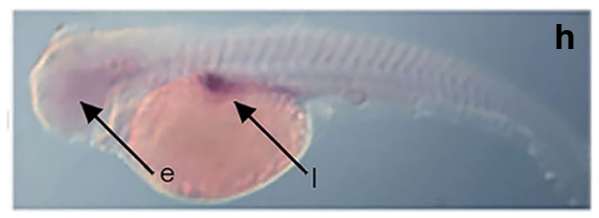

Surface
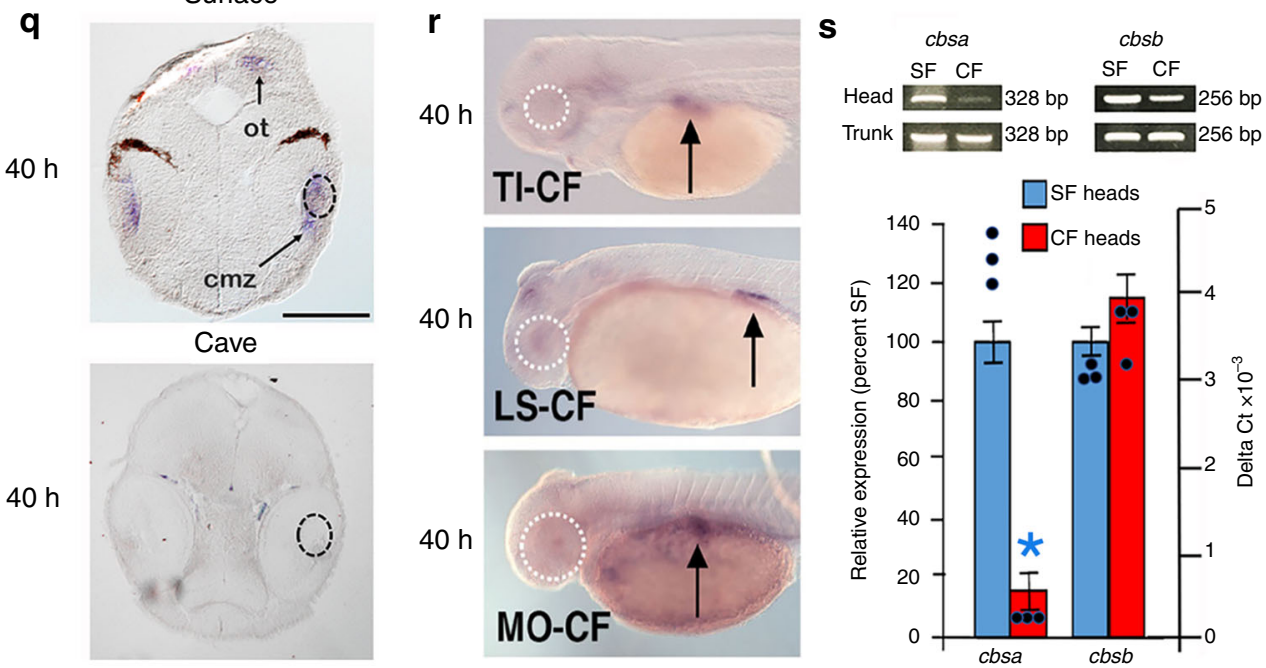

Fig. 2 Expression of $\boldsymbol{c b s a}$ and $\boldsymbol{c b s b}$ genes during Astyanax development. a-p In situ hybridization showing cbsa (a-h) and cbsb (i-p) expression in SF and PACF embryos and larvae. b brain, e eyes, $h$ heart $p$ pectoral fin buds, I liver, m myotomes. $n=500$ and at least 10 samples in each frame. $\mathbf{q}$ Sections through the heads of SF and PA-CF in situ hybridized larvae shown in $\mathbf{g}$ and $\mathbf{h}$, respectively. ot optic tectum, cmz ciliary marginal zone of retina. Dotted lines: lenses. Top of frame is dorsal. $\mathbf{r}$ In situ hybridization showing cbsa expression in TI-CF, LS-CF, and MO-CF larvae at 40 hpf. Arrows: liver. Scale bar in a is $200 \mu \mathrm{m} ;$ magnification is the same in a-f and $\mathbf{i}-\mathbf{n} . n=140,220,240,24$, and 29 from top to bottom. Scale bar in $\mathbf{g}$ is $250 \mu \mathrm{m}$; magnification is the same in $\mathbf{g}$, $\mathbf{h}, \mathbf{0}, \mathbf{p}, \mathbf{r}$. Scale bar in $\mathbf{q}$ is $300 \mu \mathrm{m}$; magnification is the same in both frames. $\mathbf{s}$ Qualitative (top) and quantitative RT-PCR comparing the levels of cbsa and cbsb expression in PA-CF heads and trunks. Graph: Bars are means and SEM of CF $\Delta \Delta$ Ct values converted to percentage of SF $\Delta \Delta$ Ct values (left $y$ axis). Data points are $\Delta C t$ values (right $y$ axis). Asterisk: significance calculated from $\Delta \mathrm{Ct}$ values $(n=3)$ by unpaired Student's $t$ test $(p=0.00)$. Source data are provided in source data file. 
a

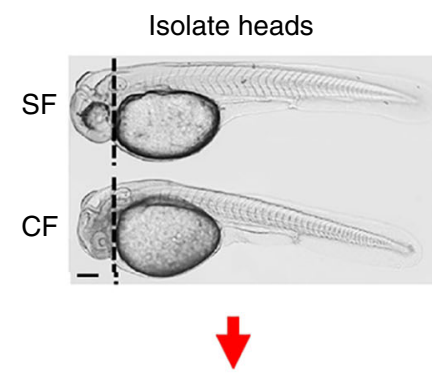

PCR cbsa and sequence PCR products

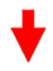

Clone and sequence $F 1$ PCR products

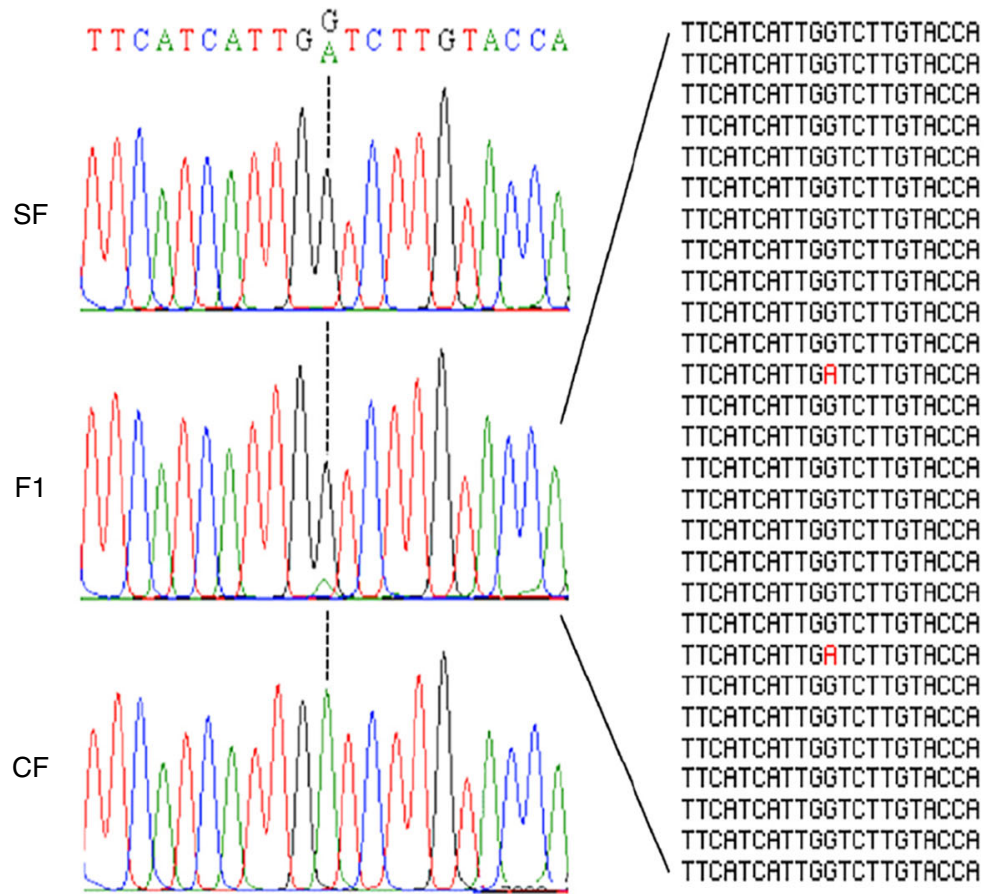

b

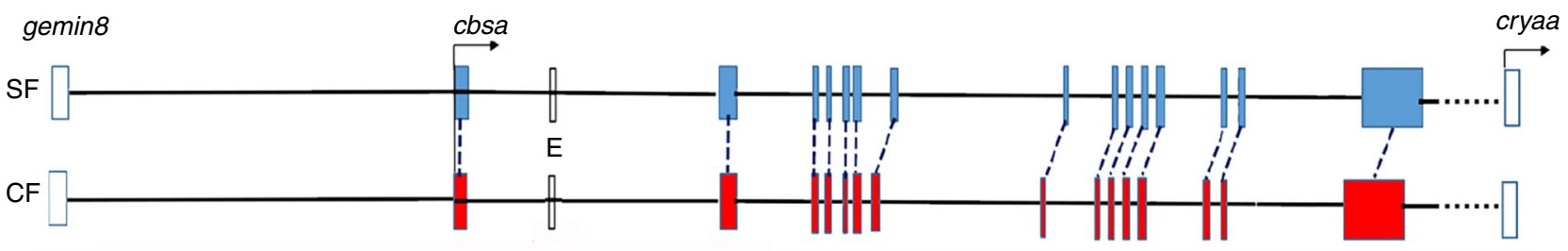

C

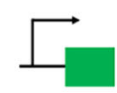

pCMV-GFP

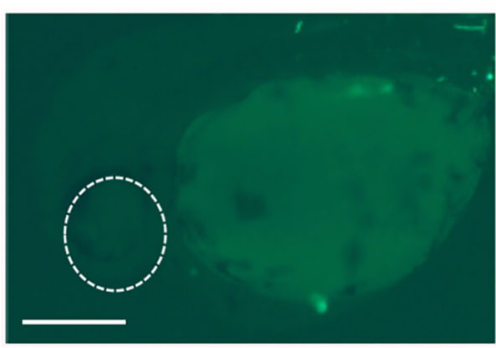

d

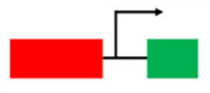

pSF-E-CMV-GFP

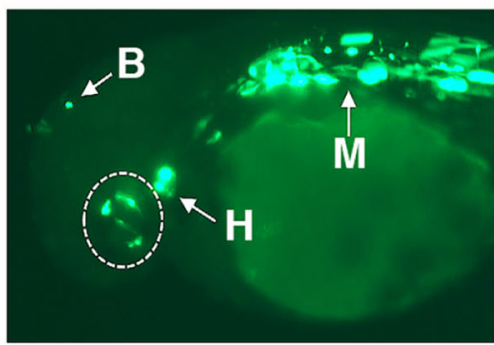

e

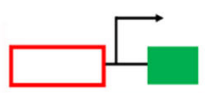

pCF-E-CMV-GFP

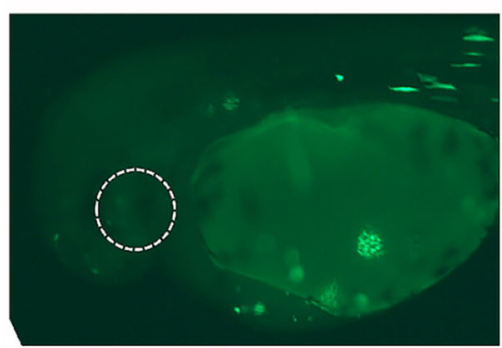

Fig. 3 Structure and cis-regulation of the Astyanax cbsa gene. a F1 hybrid test. Left: summary of procedure beginning with separation of SF, F1 hybrid, and CF larvae into heads and trunks. Scale bar: $250 \mu \mathrm{m}$. Middle: sequenced cbsa RT-PCR products from SF, F1 hybrid, and PA-CF heads showing SF and PA-CF cbsa marker SNPs (A and G nucleotides, respectively). Right: marker region sequences of 28 independently cloned RT-PCR products from F1 hybrid heads showing the PA-CF allele marker in red. b Maps of re-sequenced SF and PA-CF cbsa loci showing intron-exon organization (colored boxes) and the predicted enhancer region E. Un-colored boxes: flanking gemin8 and cryaa loci. c-e Expression of CMV-GFP constructs in SF at 40 hpf. c Control pCMVGFP construct did not drive GFP expression. $(n=49)$. d SF cbsa enhancer E containing pCMV-GFP construct drove GFP expression in eyes (enclosed by dashed lines), heart $(\mathrm{H})$, brain $(\mathrm{B})$, and myotomes $(\mathrm{M})$. $(n=22)$. e PA-CF enhancer E containing pCMV-GFP construct did not drive GFP expression. ( $n=$ 43). Dotted lines indicate eyes. Scale bar in $\mathbf{c}$ is $250 \mu \mathrm{m}$; magnification is the same in c-e. Source data are provided in source data file.

fragment containing the $\mathrm{E}$ region was fused to a cytomegalovirus (CMV) promoter and green fluorescent protein (GFP) reporter gene, injected these constructs into SF eggs, and determined reporter activity at $40 \mathrm{hpf}$ by GFP fluorescence (Fig. 3c-e). The CMV promoter alone drove modest GFP expression in SF myotomes, but no expression in the eyes or brain (Fig. 3c). In contrast, the pSF-E-CMV-GFP construct containing the SF cbsa E region drove strong GFP expression in the myotomes, eyes, heart, and the dorsal brain of $40 \mathrm{hpf}$ SF embryos but not in the liver or other locations of normal cbsa expression (Fig. 3d). Thus the $\mathrm{E}$ region contains a tissue-specific enhancer for cbsa expression. Contrastingly, the pCF-E-CMV-GFP construct 
a
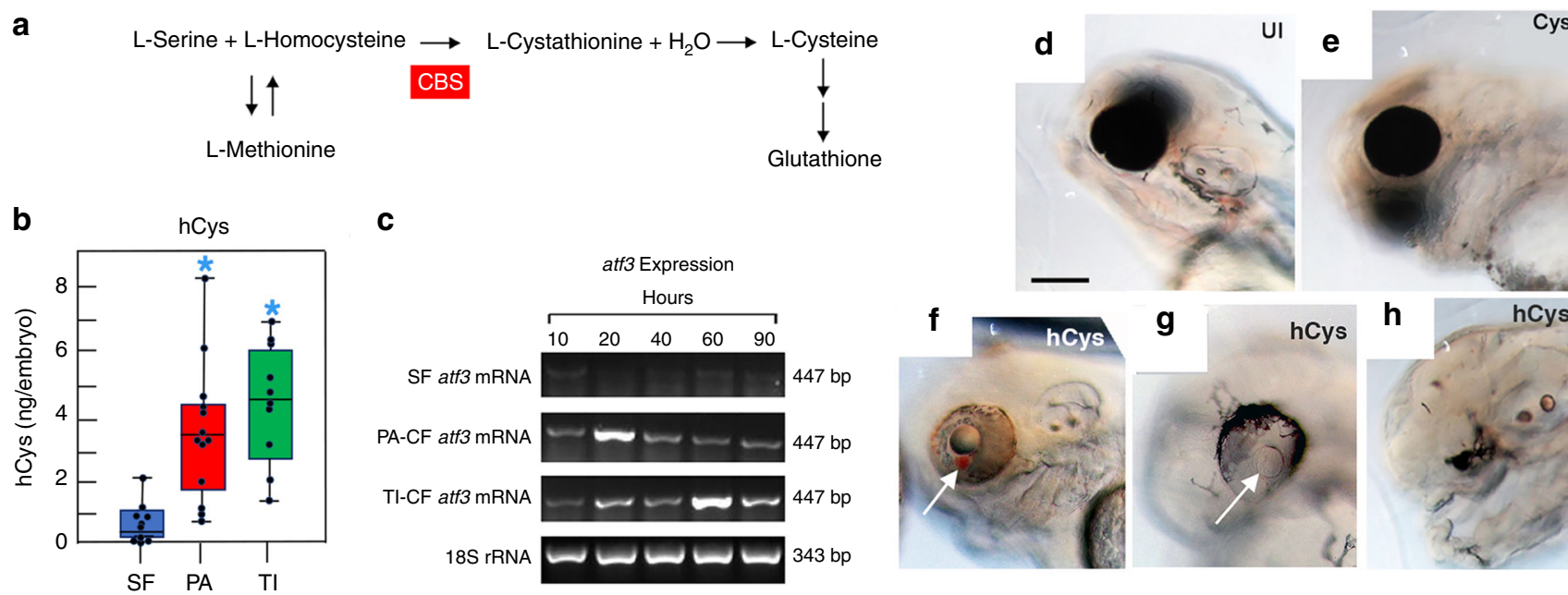

C
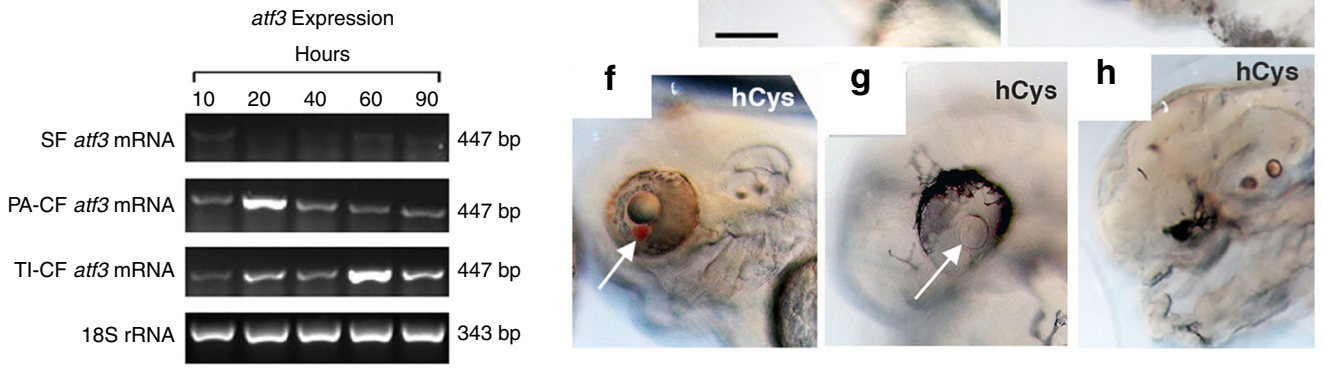

Fig. 4 Homocysteine elevation and effects on eye development. a The transsulfuration pathway showing one of the reactions catalyzed by the CBS enzyme. b ELISA determination of hCys levels in PA-CF and TI-CF relative to SF at 40 hpf. Box plots with superimposed data points of 5-7 biological replicates. Graph represents box and whiskers min to max. Asterisks: $p=0.001$ and 0.003 from left to right. Significance was determined by unpaired Student's $t$ test. c Expression of atf3 during PA-CF, TI-CF, and SF development determined by qualitative RT-PCR. Three biological replicates with similar results. d-h Effects on eye development at $4 \mathrm{dpf}$ after injection of $5 \mathrm{mM} \mathrm{hCys} \mathrm{into} \mathrm{SF} \mathrm{eggs} \mathrm{(f).} \mathrm{d,} \mathrm{e} \mathrm{Normal} \mathrm{eyes} \mathrm{in} \mathrm{un-injected} \mathrm{(UI)} \mathrm{SF} \mathrm{eggs} \mathrm{(d)} \mathrm{and} \mathrm{SF} \mathrm{eggs}$ injected with $5 \mathrm{mM}$ cysteine (Cys) (e). f-h Abnormal eyes with a hemorrhage (arrow) (f) or with a ventrally displaced lens (arrow) (g), and complete suppression of eye formation (h) in SF eggs injected with $5 \mathrm{mM}$ hCys. SF in $\mathbf{f}$ was treated with PTU to visualize hemorrhage. Scale bar: $200 \mu \mathrm{m}$; same magnification in $\mathbf{d}-\mathbf{h}$. $n$ values: see Supplementary Table 2. Source data are provided in source data file.

containing the $\mathrm{E}$ region of PA-CF cbsa drove very weak or no reporter expression in SF larval eyes, heart, and brain (Fig. 3e). These results suggest that cis-inactivating mutations in an enhancer located in the first intron are responsible for downregulation of the cbsa gene in PA-CF. However, we cannot exclude the possibility that other cis-acting changes, within or beyond the sequenced regions, may also contribute to cbsa downregulation. The indel regions in $\mathrm{E}$ contain many predicted transcription factor-binding sites that could promote restricted expression in the head and mediate changes in $c b s a$ expression in CF and SF (Supplementary Fig. 3). These results (and functional data below) suggest that cbsa could be one of the mutated genes responsible for CF eye degeneration.

CF show homocystinuria-like features. The cbsa gene encodes CBS, the limiting enzyme of the transsulfuration pathway, which converts hCys to cystathionine, a precursor of cysteine and glutathione $^{22}$ (Fig. 4a). Mutations in the human $c b s$ gene are the major cause of homocystinuria, a disorder in methionine metabolism resulting in toxic hCys accumulation and eye abnormalities $^{23,24}$. In fact, the small eyes of CF larvae resemble the eyes of some homocystinuria patients in showing an "off-center" lens ${ }^{2-5}$. To test the possibility that CF show homocystinuria-like features, we compared hCys levels by enzyme-linked immunosorbent assay (ELISA) quantification and expression of activating transcription factor 3 (atf3), a major hCys-responsive gene ${ }^{25}$, in CF and SF larvae. The results showed increases of hCys levels in PA$\mathrm{CF}$ and TI-CF larvae at $40 \mathrm{hpf}$ (Fig. $4 \mathrm{~b}$ ) and upregulation of the atf3 gene in PA-CF and TI-CF relative to SF from about 1 to 4 days post-fertilization (dpf) (Fig. 4c), indicating that hCys accumulates in CF. To examine the developmental effects of increased hCys, we injected hCys into SF eggs and examined eye morphology at $40 \mathrm{hpf}$. The results showed that about $23 \%$ of the larvae that developed from SF eggs injected with hCys showed defects in eye formation, including reduced eye size, displacement of the lens to the ventral side of the retina, or complete eye loss (Fig. 4d-h; Supplementary Table 2), and in some cases hemorrhages occurred in or near the eyes (Fig. 4f; and see below). In contrast, only about $4 \%$ of control larvae that developed from eggs injected with cysteine showed effects on eye development (Fig. 4a, e; Supplementary Table 2), and no eye hemorrhages were seen in these embryos. These results indicate that CF exhibit homocystinuria-like features that could impact eye development.

Defects in CF optic vasculature. High hCys levels are known to affect cardiovascular function, increasing the risk of blood clots, aneurysms, and hemorrhagic stroke in homocystinuria patients and $\mathrm{Cbs}^{+/-}$mice ${ }^{26,27}$. Therefore, we examined optic vasculature in CF by microangiography, blood cell staining, and eye imaging (Fig. 5). The results revealed leaky optic and brain vasculature, erythrocyte pooling and aneurysms around the eyes, and eye hemorrhages (Fig. 5a-j), which were observed in up to $20-40 \%$ PA-CF, TI-CF, and LS-CF larvae (Fig. 5l) but not in $>1000$ SF larvae (Fig. 5f). The eye hemorrhages were generally unilateral, ranging from small foci on the dorsal side of the eye to large areas of the orbit and in most cases persisted for only about 3-4 days (Fig. 5l). During this period, the degenerating CF eyes were invaded by phagocytic macrophages, probably to remove leaked blood cells (Fig. 5k). Survival studies showed that PA-CF larvae with eye hemorrhages had similar viability to SF larvae (Fig. $5 \mathrm{~m}$ ), showing that defective optic vasculature does not affect CF viability. The results indicate that defects in optic vasculature accompany CF eye degeneration.

Role of $\boldsymbol{c b s}$ genes in eye and optic vasculature development. To address the roles of $c b s$ genes in eye and vasculature development, we knocked down $c b s a$ and $c b s b$ expression by injecting SF eggs with splice-inhibiting morpholinos (MOs) (Fig. 6a-l). To validate this approach, we amplified and sequenced PCR products from the MO-targeted regions of $c b s$ morphants and obtained products of two different sizes: one with sequence corresponding to the processed $c b s a$ exons 5 and 6 or $c b s b$ exons 3 and 4 , and the other with sequence including the unprocessed introns containing the expected translation stop sites (Supplementary Fig. 4a-f). The cbsa and $c b s b$ MOs were specific for the corresponding genes, respectively (Supplementary Fig. 4a, b). 

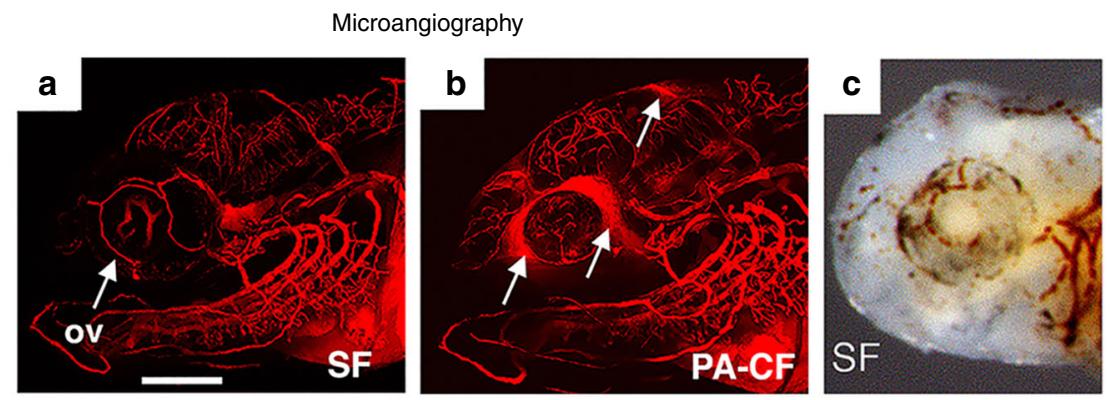

Erythrocyte staining
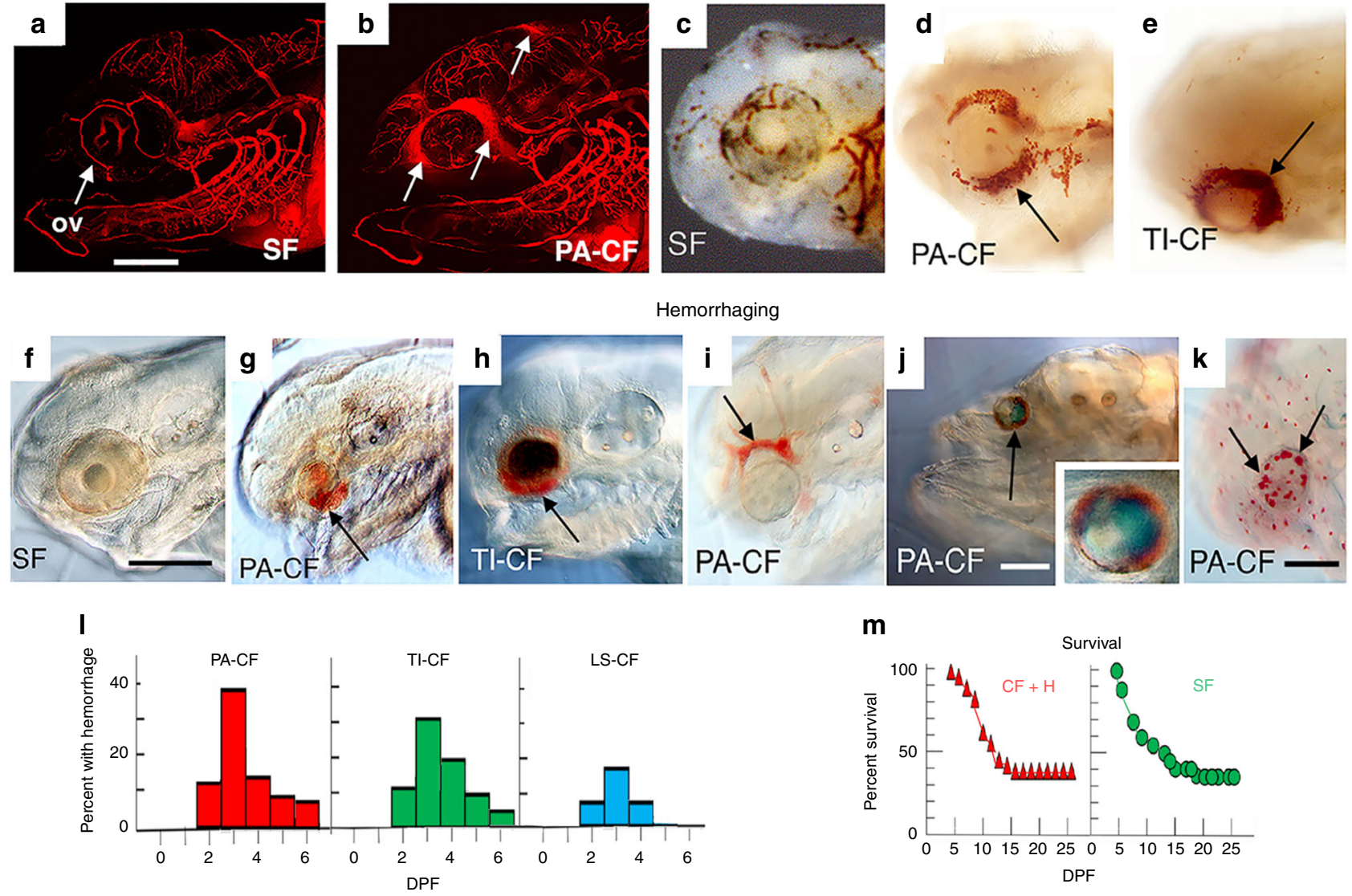

Fig. 5 Defective optic vasculature in cavefish. a, b SF (a) and PA-CF (b) at 5 days post-fertilization (dpf) showing leaky vasculature around the PA-CF eye (arrows). ov optic vasculature. Each microangiography assay was conducted with three biological replicates. c-e SF (c), PA-CF (d), and TI-CF (e) showing stained erythrocytes (arrows in d, e) pooled around CF eyes at $2.5 \mathrm{dpf} . n=24$ for SF and 36 for PA-CF. f-h SF (f), PA-CF (g), and TI-CF (h) at 4 dpf showing hemorrhages (arrows in $\mathbf{g}, \mathbf{h}$ ) in CF eyes. i Aneurysm (arrow) in PA-CF optic vasculature at 4 dpf. $\mathbf{j}$ PA-CF showing eye hemorrhage (arrow) at 14 $\mathrm{dpf}$ (inset, 2.5 magnification of eye area). $\mathbf{k}$ Neutral red staining of PA-CF at $7 \mathrm{dpf}$ showing macrophages (arrows) in degenerating eyes ( $n=36$ larvae). Scale bars: a $300 \mu \mathrm{m}$ (same magnification in $\mathbf{a}, \mathbf{b}), \mathbf{f} 200 \mu \mathrm{m}$ (same magnification in $\mathbf{c}-\mathbf{i}), \mathbf{j}, \mathbf{k} 150 \mu \mathrm{m}$. I The percentage of PA-CF $(n=2101), \mathrm{TI}-\mathrm{CF}(n=$ 268), and LS-CF $(n=121)$ with hemorrhages during larval development. $\mathbf{m}$ Survival of SF (right) $(n=100)$ and PA-CF $(n=46)$ with hemorrhages (left) during larval development. Source data are provided in source data file.

We next determined the effects of cbsa and cbsb MO knockdown on eye development. About $40 \%$ of cbsa morphants developed morphologically abnormal eyes: a small proportion of these completely lacked eyes and the remainder had reduced eyes compared to those injected with control $\mathrm{MO}$, including ventrally displaced lenses with decreased expression of the lens marker gene cryaa (Fig. 6a, b; Supplementary Table 3; Supplementary Fig. $4 \mathrm{~g}, \mathrm{~h}$ ), which is similar to the CF eye phenotype $\mathrm{e}^{2-5}$. About $82 \%$ of the $c b s b$ morphants lacked eyes and cryaa expression, whereas the remainder had reduced eyes compared to controls and showed ventrally displaced lenses (Fig. 6a, c; Supplementary Table 3; Supplementary Fig. 4g, h). No other morphological changes were noted in these $c b s$ morphants (Supplementary Fig. 4g). The control morphants showed about $97 \%$ normal optic phenotypes (Fig. 6a; Supplementary Table 3; Supplementary Fig. 4g, h). Co-injection of $c b s a$ and $c b s b$ MOs generated eye phenotypes resembling the single cbsb MO injections (Supplementary Table 3 ). We also tested whether the morphant eye phenotypes could be rescued by injection with SF cbsa mRNA. The results showed that about $85 \%$ of $c b s a$ mRNA-injected $c b s a$ morphants developed morphologically normal eyes, compared to about 30\% with normal eyes in cbsa morphants that were not injected with cbsa mRNA (Fig. 6j; Supplementary Table 4). Furthermore, the proportion of $c b s a$ mRNA-injected $c b s b$ morphants lacking eyes was reduced to $30 \%$, compared to $75 \%$ in $c b s b$ morphants that were not injected with $c b s a$ mRNA, although normal eyes were not obtained in the cbsa mRNAinjected $c b s b$ morphants (Fig. 6j; Supplementary Table 4). These results further support the specificity of $c b s$ MO effects.

To determine whether the CF eye degeneration phenotype is reversible, SF cbsa mRNA was injected into 1-cell PA-CF embryos and evaluated for eye morphology and lens apoptosis at $5.5 \mathrm{dpf}$ (Fig. $6 \mathrm{~m}-\mathrm{r}$ ). Although no increases in mean eye size were observed between the cbsa mRNA-injected and control larvae (Fig. $6 \mathrm{~m}$ ), the cbsa mRNA-injected larvae showed a significant increase in average lens size compared to controls (Fig. 6n). Importantly, lens apoptosis, a key feature of CF eye degeneration ${ }^{2,4}$, was substantially reduced or not observed in the cbsa mRNA-injected PA-CF larvae, whereas the control PA-CF larvae showed typical lens apoptosis, and no differences in retinal apoptosis were detected between cbsa mRNA-injected and control CF larvae (Fig. 6q, r). Other aspects of the eye degeneration phenotype, such as the formation of optic hemorrhages and the invasion of macrophages, were not altered by cbsa mRNA injection (Fig. 6o, p). The results indicate that $c b s a$ overexpression can partially reverse $\mathrm{CF}$ eye degeneration.

We also examined the effects of $c b s$ MO knockdown on hCys accumulation and optic vasculature development. ELISA 

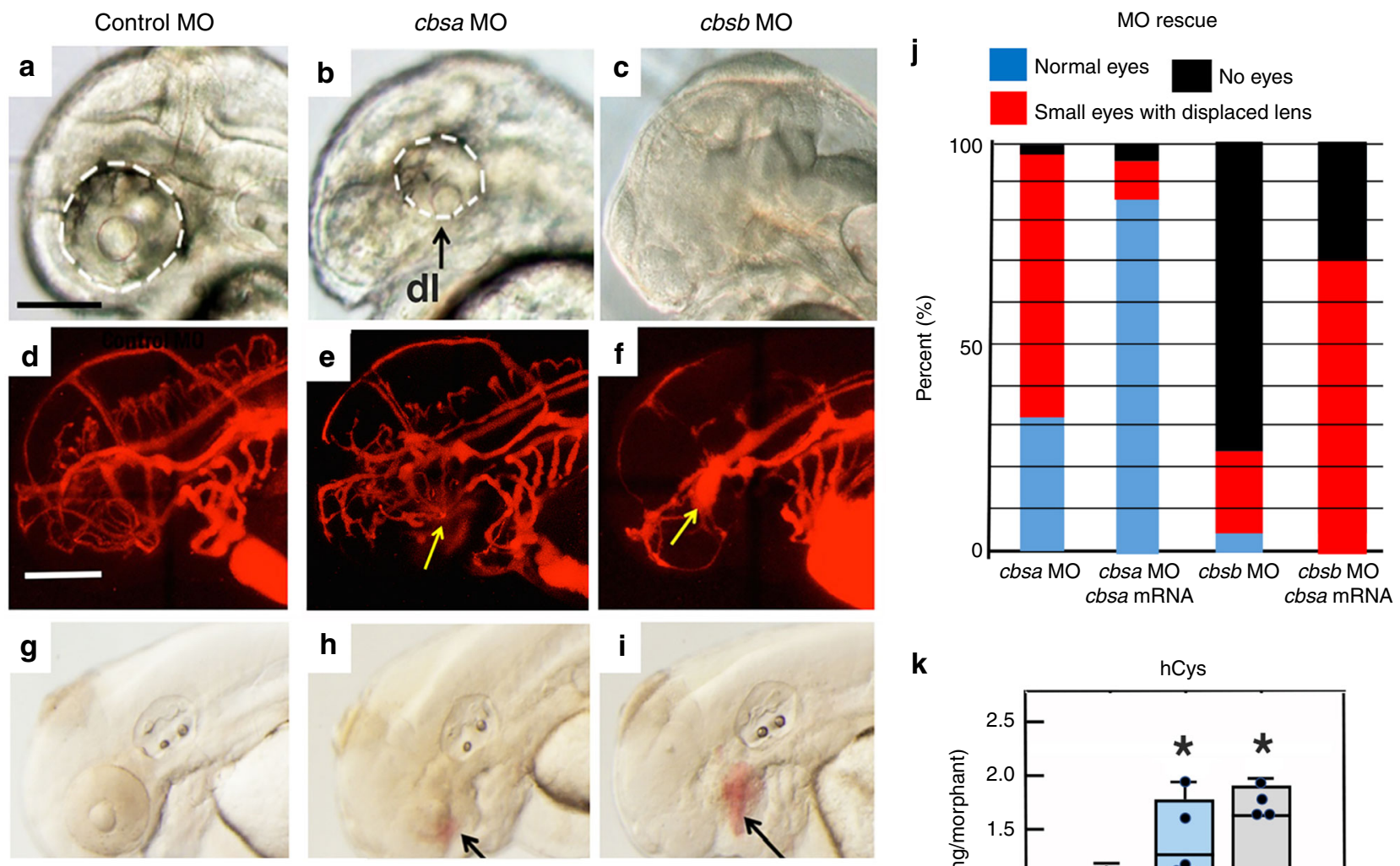

m

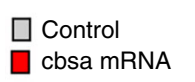

cbsa mRNA cavefish rescue
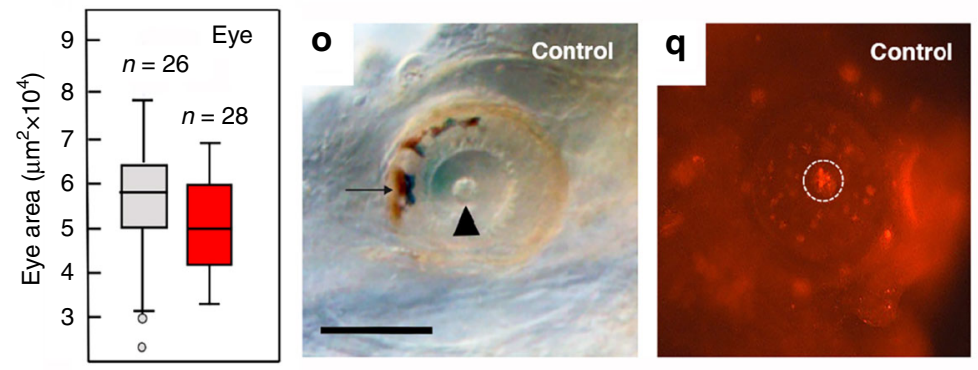

$\mathbf{k}$

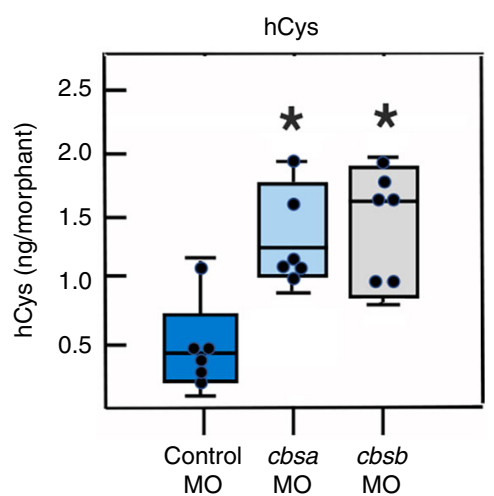

n
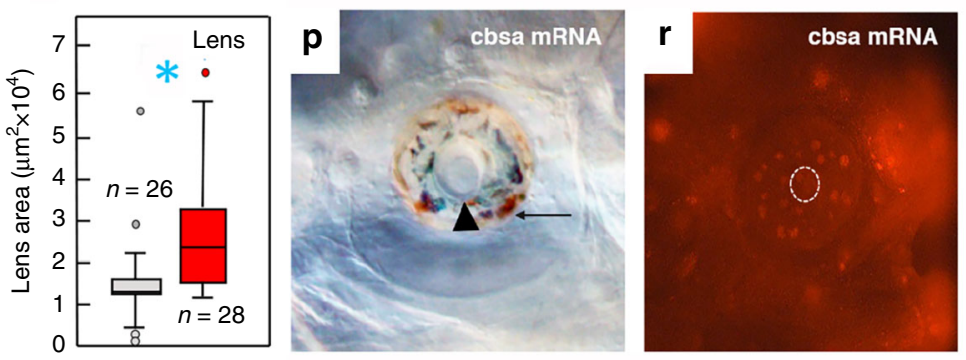

I

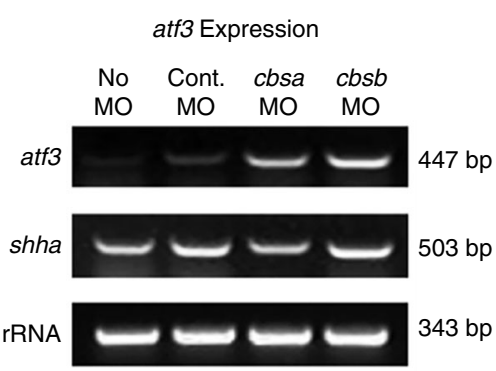

Fig. 6 Effects of cbs gene knockdown and rescue by cbsa overexpression. a-i Morpholino (MO) knockdown of cbsa and cbsb genes. Effects of control $(\mathbf{a}, \mathbf{d}, \mathbf{g})$, cbsa $(\mathbf{b}, \mathbf{e}, \mathbf{h})$, and cbsb (c, $\mathbf{f}, \mathbf{i})$ MOs on eye development (a-c), optic vasculature integrity determined by microangiography (d-f), and optic hemorrhage formation (g-i) in SF at 40 hpf. Each assay was conducted with three biological replicates. Dashed lines: eyes. Yellow arrows: leaky vasculature. dl displaced lens, h hemorrhages. Scale bar in a is $200 \mu \mathrm{m}$; magnification is the same in $\mathbf{a}-\mathbf{i}$. $\mathbf{j}$ Partial reversal of cbsa MO- and cbsb MO-induced eye defects in SF morphants by injection of cbsa mRNA. Bar graphs show the percentage of normal and abnormal eye phenotypes. See Supplementary Table 4 for $n$. Significance by chi-square test. Chi ${ }^{2}$ is 129.9987; $p<0.001$. k, I Effects of cbs MO knockdown on hCys levels (k) and atf3 and shha gene expression (I) in SF at $40 \mathrm{hpf}$. $\mathbf{k}$ Box plots with superimposed data points of six biological replicates. Significance was determined by unpaired Student's $t$ test. ( $p=0.006$ and 0.003 from left to right). $\mathbf{m}-\mathbf{r}$ Partial rescue of eye phenotypes by SF cbsa mRNA injection in PA-CF embryos. $\mathbf{m}, \mathbf{n}$ Box plots with superimposed data points showing mean eye $(\mathbf{m})$ and lens $(\mathbf{n})$ sizes at $6.5 \mathrm{dpf}$ in control (o) and mRNA injected PA-CF embryos (p). $n$ is shown above or below boxes. Significance was determined by unpaired Student's $t$ test. $(p=0.003)$. o, $\mathbf{p}$ Eyes from $\mathbf{m}(\mathbf{0})$ and $n$ ( $\mathbf{p}$ ) showing macrophages (arrows). Arrowhead: lens. q, $\mathbf{r}$ Lens apoptosis in control (q) but not in mRNA injected (r) PA-CF embryos. Dashed lines: lens. $n=22$ for CF and 30 for SF. Asterisks: Significance was determined by unpaired Student's $t$ test. $(p=0.006)$. Scale bar in $\mathbf{o}$ is $350 \mu \mathrm{m}$; magnification is the same in $\mathbf{0}-\mathbf{r}$. Graphs represent box and whiskers min to max. Source data are provided in source data file. 


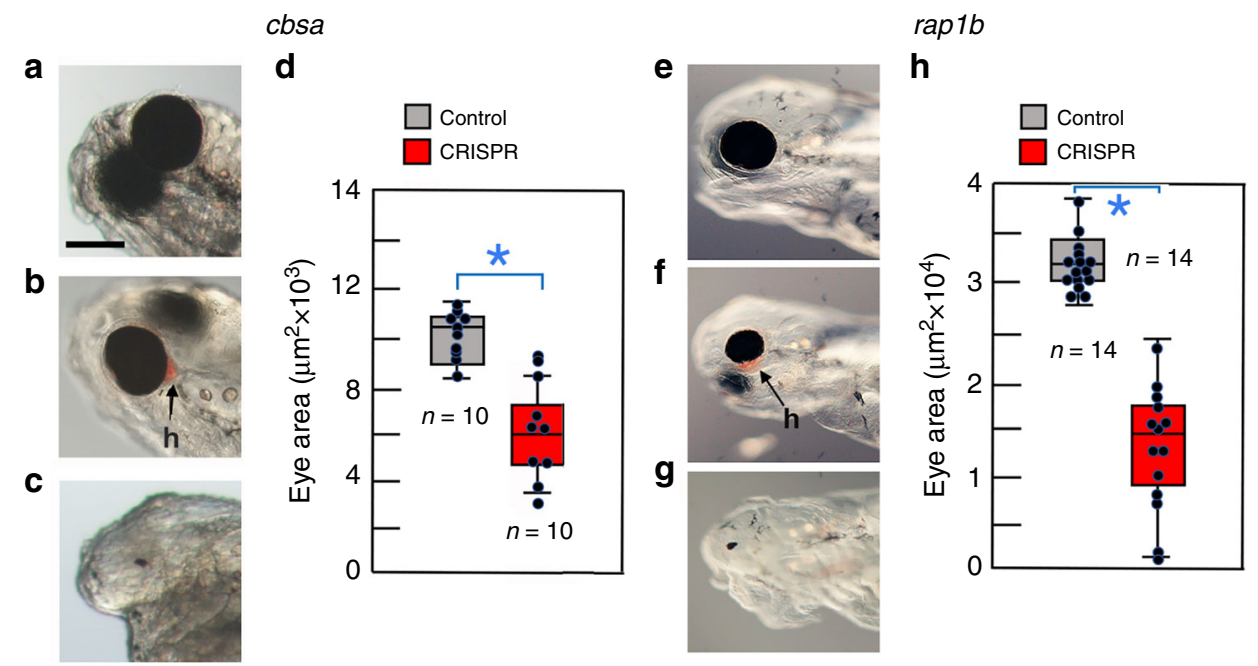

Fig. 7 Effects of $\boldsymbol{c b s a}$ and rap1b gene editing on eye development. $\mathbf{a}-\mathbf{c}, \mathbf{e}-\mathbf{g}$ Control $(\mathbf{a}, \mathbf{e})$ and CRISPR-Cas9-edited $(\mathbf{b}, \mathbf{c}, \mathbf{f}, \mathbf{g})$ larvae showing the range of eye phenotypes. $\mathbf{d}, \mathbf{h}$ Box plots comparing mean eye size in control and CRISPR-edited larvae at $3.5 \mathrm{dpf}$. Graphs represent box and whiskers min to max. $n$ is shown above or below each box. Error bars: SEM. Asterisks indicate significance in unpaired Student's $t$ test. $(p=0.000$ in $\mathbf{d}, \mathbf{h}) . n$ is shown near box plots. Scale bar in $\mathbf{a}$ is $250 \mu \mathrm{m}$; magnification is the same in $\mathbf{a}-\mathbf{c}$ and $\mathbf{e - g}$. Source data are provided in source data file.

quantification showed that hCys levels were increased in $c b s a$ and cbsb morphants compared to controls (Fig. 6k), and increased atf3 expression, but not the control sonic hedgehog a (shha) gene, was also observed in cbs morphants (Fig. 61). These results show that $c b s$ knockdown increases hCys levels. To determine the effects on vasculature development, we conducted microangiography of $c b s$ and control MO-injected larvae. The $c b s$ morphants showed leaky vasculature around the developing eyes and in the brain (Fig. 6e, f), whereas vascular integrity was not compromised in control morphants (Fig. 6d). Moreover, imaging indicated that $c b s a$ and $c b s b$ morphants developed hemorrhages around the eyes (Fig. 6h, i), but hemorrhages were not observed in control morphants (Fig. $6 \mathrm{~g}$ ). These results demonstrate that the hCys and optic vasculature phenotypes of CF can be generated in SF by $c b s$ gene knockdown. The effects of cbsa MO knockdown on SF eye development were confirmed by clustered regularly interspaced short palindromic repeats (CRISPR)-Cas9 editing of the $c b s a$ gene (Fig. 7a-d; Supplementary Fig. 5a). The cbsa exon 2 was targeted by two guide RNAs, and the mosaic F0 larvae were scored for eye phenotypes. The cbsa-edited larvae showed eye abnormalities ranging from reduced eyes with hemorrhages to completely absent eyes (Fig. 7a-d). In summary, the MO knockdown and CRISPR-Cas9 editing results show that blocking cbs gene expression increases hCys levels, induces defects in optic vasculature, and generates abnormal eye morphology, suggesting a role for the circulatory system in CF eye degeneration.

Inhibition of circulatory function affects eye growth. To confirm the role of the circulatory system in CF eye degeneration, we used CRISPR/Cas9 to edit the raplb gene in SF and determined the effects on eye size and morphology in the F0 generation (Fig. 7e-h; Supplementary Fig. 5b). The rap1b gene is required in endothelial cells for vascular integrity and in mutant form shows cranial hemorrhages in zebrafish ${ }^{28}$, resembling the Astyanax CF and SF $c b s$ knockdown phenotypes. The rap1b-edited and control larvae showed eyes of similar size at $2.5 \mathrm{dpf}$, but by $3.5 \mathrm{dpf}$ eye size was much smaller in the CRISPR-edited larvae than in the control larvae, and in some cases eye hemorrhages were formed or eyes were missing (Fig. 7e-h). These results suggest that interference with circulatory system function in rap1b mutants can phenocopy the effects of hypomorphic cbsa, confirming the conclusion that defective optic vasculature is an important regulator of CF eye degeneration.

Degenerating CF eyes exhibit hypoxia-related stress. The discovery of defective optic vasculature opened the possibility that CF eyes may be subject to hypoxic stress. To explore this possibility, the Astyanax homologs of some of the genes responsive to hypoxia-related stress in zebrafish ${ }^{29,30}$ were identified, and their expression levels were compared in SF and PA-CF larvae that were separated into heads and trunks at $36 \mathrm{hpf}$ (Fig. 8a, b). We selected the hypoxia-inducible factor $1 \alpha a$ (hif1 $\alpha a$ ) and hif1 $\alpha b$, which encode master regulators of hypoxia ${ }^{31}$ (but note that HIFa can also be induced by other types of stress); hemopexin ( $h p x)$, which encodes a major hemolysis-sensitive heme scavenger ${ }^{32}$; myoglobin $(\mathrm{mb})$, which encodes an oxygen transporter in muscle cells ${ }^{33}$; and oxidative stress growth inhibitor 1 (osgn1), which encodes an oxidative stress-response protein that regulates cell death, as examples of genes upregulated by hypoxia in zebrafish. We also selected the rps $3 a$ gene, encoding a ribosomal protein that is upregulated by hypoxic stress in the liver of another teleost $^{34}$. The results showed that the hif1 $\alpha a$, hifl $\alpha b$, and $m b$ genes were upregulated in the head compared to the trunk of PA-CF larvae (Fig. 8a). Furthermore, $r p s 3 a$ was upregulated in the PACF trunk, where the liver is located, compared to the head (Fig. 8a). No significant differences were seen in the expression of these genes between the head and trunk of SF larvae (Fig. 8a). The results suggest that the expression of genes related to hypoxic stress are increased in CF heads. To investigate hypoxic stress in degenerating $\mathrm{CF}$ eyes, we stained $\mathrm{CF}$ and $\mathrm{SF}$ controls with an antibody against the master hypoxia-regulating factor HIFla. Early in development, when both SF and PA-CF eyes increase in size, HIFla was not detected in SF or PA-CF eyes, but as optic development proceeded and CF eye growth was arrested, HIF1a was seen in CF, but not in SF eyes, and HIF1a continued to be present through the subsequent eye degeneration (Fig. $8 \mathrm{~b}-\mathrm{d}$ ). These results suggest that degenerating CF eyes show hallmarks of hypoxic stress.

Optic vasculature disruption is independent of lens apoptosis. Previous studies showed that lens apoptosis is an early mediator of $\mathrm{CF}$ eye degeneration ${ }^{2-5}$. Because apoptosis and reduced lens 
a
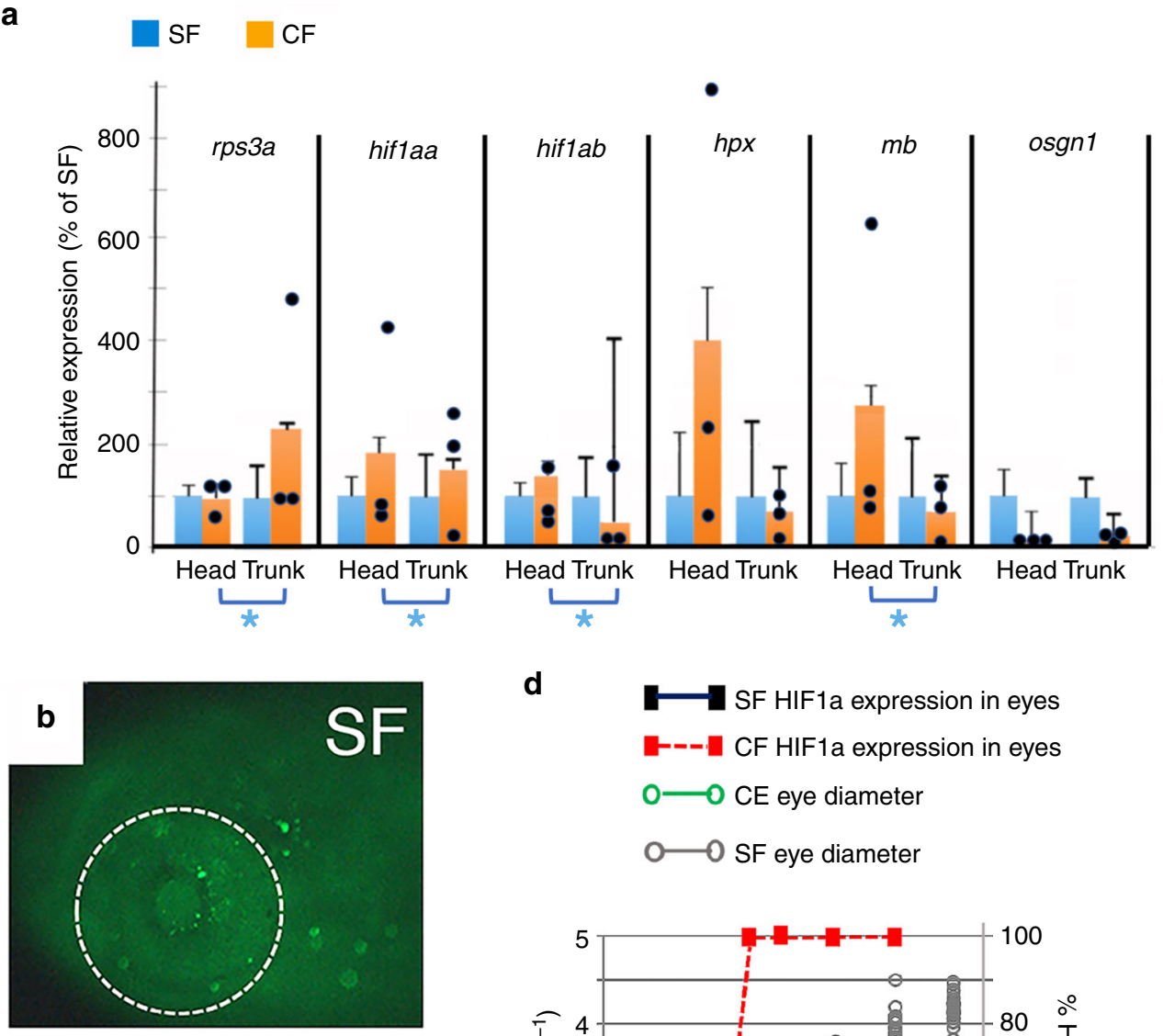

d
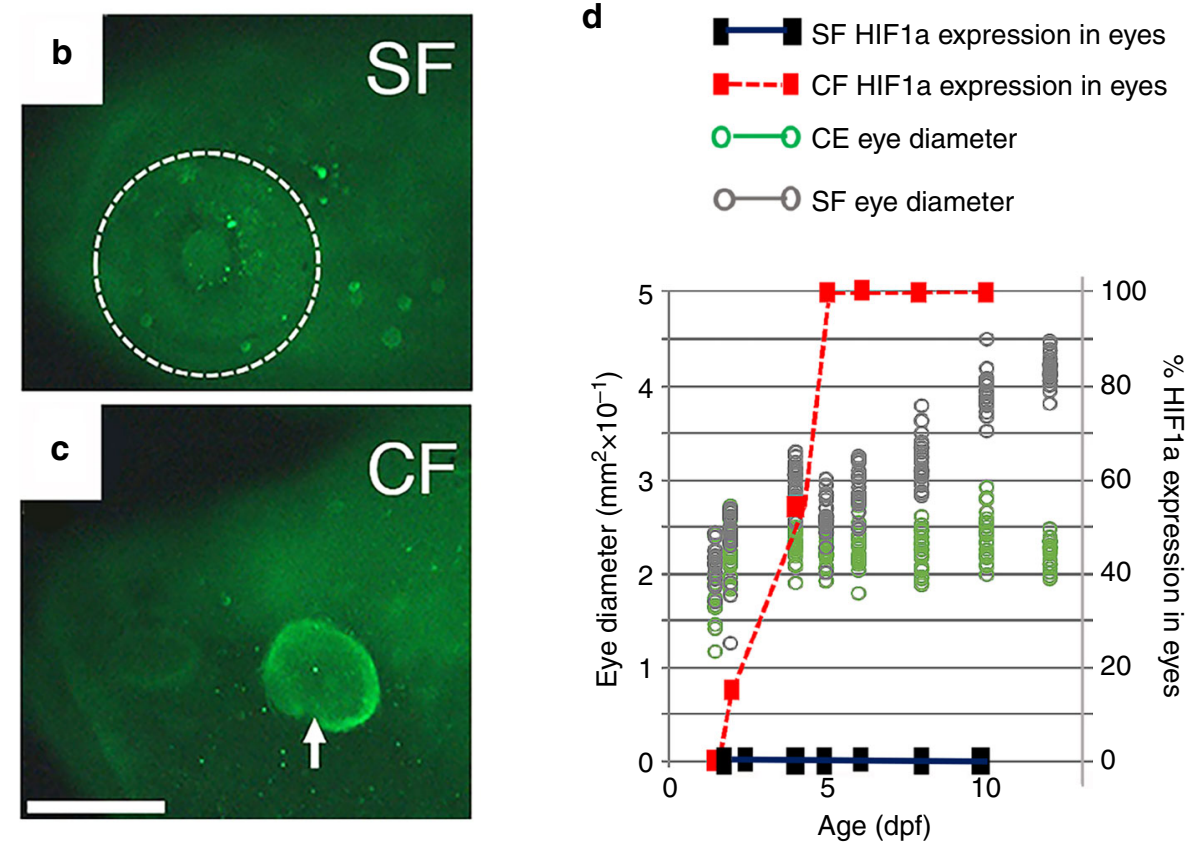

Fig. 8 Hypoxia in cavefish. a Expression of hypoxia-sensitive genes by QPCR in isolated SF and PA-CF heads and trunks. Bars are means and SEM of CF $2^{-\Delta \Delta \mathrm{Ct}}$ values converted to percentage of SF values in three biological replicates. Data points are $\Delta \Delta \mathrm{Ct}$ values $(n=3)$. Asterisks: significance calculated from $\Delta \Delta$ Ct values by unpaired Student's $t$ test ( $p=0.000,0.002,0.002$, and 0.002 from left to right). b, c Determination of hypoxia-inducing factor $1 \alpha$ (HIF1a) expression in SF (b) and PA-CF (c) eyes at $7 \mathrm{dpf}$ by immunostaining. Dotted line surrounds SF eye. Arrow indicates the margin of CF eye. SF: $n=$ 16. PA-CF: $n=16$. Scale bar: $150 \mu$ m; same magnification in $\mathbf{b}$ and $\mathbf{c}$. d HIF1 $\alpha$ expression in CF eyes determined by immunostaining compared to optic growth during SF and PA-CF development. $n=16$ larvae for each time point. Source data are provided in source data file.

size in CF could be partially rescued by overexpression of cbsa in CF (Fig. 6m-r), we conducted additional experiments to understand the relationship between CF lens dysfunction, cbsa downregulation, and optic vasculature defects. First, we assayed lens apoptosis in $c b s$ morphants and CRISPR-Cas9-edited SF and CF control larvae at $40 \mathrm{hpf}$. Apoptotic cells were detected in the lens of CF controls (Fig. 9b) but not in the lens of SF controls (Fig. 9a) or in the lenses of $c b s a$ and $c b s b$ morphants or $c b s a$ CRISPRCas9-edited larvae (Fig. 9c, d, f), showing that interference with $c b s$ function does not promote lens apoptosis. Second, we removed a lens from eyes on one side of the SF larval head at 30 hpf and asked whether this operation induced the formation of optic hemorrhages later in development. The results showed that optic hemorrhages were not induced in SF larvae lacking a single lens (Fig. 9g). Together, these results suggest that lens dysfunction and hypomorphic cbsa-mediated optic vasculature defects are independent events in CF eye degeneration.

\section{Discussion}

In the present study, we have identified cbsa as one of the genes responsible for eye degeneration in A. mexicanus CF. Furthermore, studies of the hypomorphic cbsa phenotype suggest a mechanism for eye degeneration based on the disruption of optic circulatory system function. Our work builds on previous studies that determined the multiplicity of genetic factors involved in $\mathrm{CF}$ eye loss ${ }^{1,2,10}$, identified QTL and corresponding genomic regions responsible for the regressive $\mathrm{CF}$ eye phenotype ${ }^{11-13,15}$, and 
cbs Morpholino

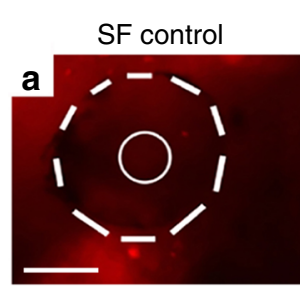

SF cbsa MO

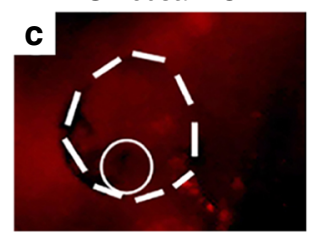

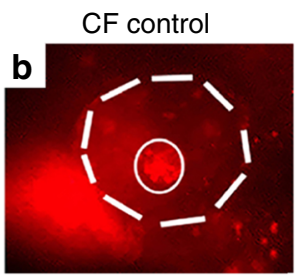

$\mathrm{SF} c b s b \mathrm{MO}$

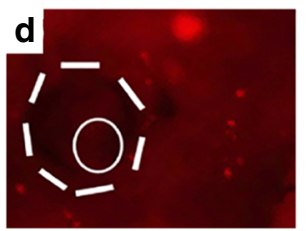

cbsa CRISPR

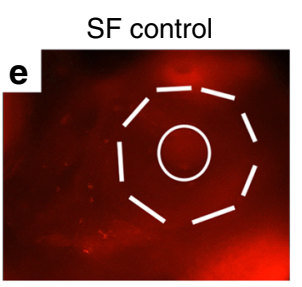

SF CRISPR

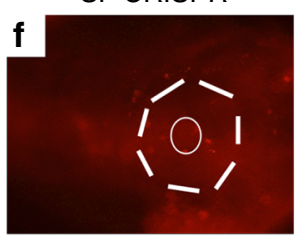

Lens removal
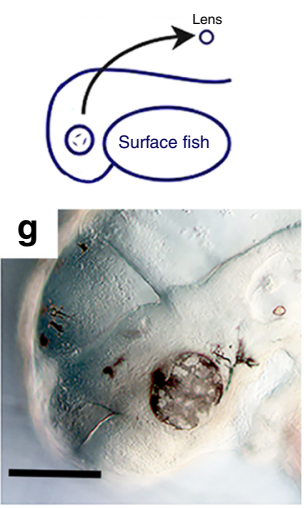

Fig. 9 The expression of $\boldsymbol{c b s}$ genes and lens-associated phenotypes. a-f Apoptosis detected by Lysotracker staining in cbs morphant larvae and controls at 68 hpf. a SF control with no lens apoptosis $(n=14)$. b PA-CF control with lens apoptosis. $(n=26)$. cbsa (c) and cbsb (d) SF morphants with no lens apoptosis. ( $n=22$ and 14, respectively). e, $\mathbf{f}$ Apoptosis detected by Lysotracker staining in cbsa CRISPR-Cas9-edited SF larvae at 60 dpf: e SF control with no apoptosis $(N=24) ; \mathbf{f}$ SF CRISPR-Cas9 with no lens apoptosis $(n=30)$. Dashed lines: outline of eyes. Complete lines: outline of lenses. $\mathbf{g}$ Lens removal from the optic cup on one side of SF larvae at $30 \mathrm{hpf}$ (illustration of operation shown on top) did not induce optic hemorrhages at $4 \mathrm{dpf}$. ( $n=23$ ). Scale bar in a: $100 \mu \mathrm{m}$; Magnifications are the same in a-f. Source data are provided in source data file.

tested candidate genes for mutations and roles in $\mathrm{CF}$ eye degeneration ${ }^{17}$. However, none of the mutated genes responsible for CF eye loss were identified.

Our study, along with the studies mentioned above, provides multiple lines of evidence suggesting that $c b s a$ is one of the mutated genes responsible for CF eye degeneration. The cbsa gene is located near the peak marker of a PA-CF eye size QTL ${ }^{11,15}$, was recognized as a potential candidate for controlling CF eye loss by Ingenuity Pathway Analysis of gene expression data ${ }^{15}$, and is downregulated in eyes during larval development in four different CF populations: PA-CF, LS-CF, TI-CF, and MO-CF. Importantly, two or more of these CF populations are thought to have evolved the eyeless phenotype independently and share some of the same genetic factors involved in eye loss ${ }^{13}$. Our results suggest that $c b s a$ may be one of the shared genes. We have also presented MO knockdown and CRISPR-Cas9 gene-editing results showing that the cbsa gene is required for SF eye development. When cbsa function was disrupted, eye formation was completely abolished or abnormal eyes with ventrally displaced lenses-a part of the typical CF larval eye phenotype $\mathrm{e}^{2-5}$-were formed without affecting general larval morphology. Our results are consistent with the possibility that the hypomorphic cbsa gene is responsible for the phenotypic effects represented by an Astyanax eye QTL. However, more functional testing of other genes within the genomic interval will be needed to conclude that it is the only gene in this QTL that is mutated and involved in eye degeneration. Although the lens degeneration phenotype was partially reversed by overexpressing cbsa in CF embryos, there was no rescue of eye size or hemorrhage formation, indicating that $c b s a$ does not have the power to reverse the entire CF eye degeneration phenotype. In this regard, it is important to note that $c b s a$ is only one of many genetically ${ }^{10-13,15}$ or epigenetically ${ }^{14}$ modified genes that may regulate $\mathrm{CF}$ eye degeneration and that other genes remain to be discovered to fully understand this complex phenotype.

Our studies of the CF cbsa phenotype revealed that A. mexicanus CF show similarities to the human disease homocystinuria and mouse Cbs models of this disease with respect to eye abnormalities, elevated hCys levels, and deficiencies in the cardiovascular system ${ }^{23-27}$. We found approximately 3-4-fold increases in hCys in PA-CF and TI-CF larvae relative to SF larvae.
Heterozygous $\mathrm{Cbs}^{+/-}$mice show a 2-fold elevation of hCys levels, which results in alterations of retinal vasculature, and a 40 -fold increase in hCys causes death in utero in $\mathrm{Cbs}^{-1-}$ mice $26,27,35$. The ability of CF to survive mild homocystinura-like effects and develop into healthily adults is likely due to compensation for cbsa downregulation by the paralogous $c b s b$ gene. The Astyanax $c b s a$ and $c b s b$ genes do not appear to be co-expressed in the eyes, which is the likely reason that hypomorphic cbsa effects are restricted to these organs and do not engender a lethal response. We also observed cbsa expression in the SF optic tectum, as also reported for $c b s$ genes in zebrafish ${ }^{19}$ and mouse $\mathrm{e}^{36}$, and $c b s a$ is downregulated in this region of the CF brain. The optic tecta are regressed coordinately with the eyes during CF evolution ${ }^{37,38}$. Therefore, mutated cbsa could promote the regression of CF eyes and optic tecta as a functional unit.

Mutations in $c b s$ reduce life expectancy in homocystinuria patients and are responsible for cardiovascular defects in mouse models of homocystinuria ${ }^{24-27}$. We discovered defects in optic and cranial vasculature, including vascular leakages, eye hemorrhages, and aneurysms, during the critical period of CF eye degeneration. In our study, these defects were only seen after SF eggs were injected with hCys, a cbs paralog was knocked down by MO injection, or $c b s a$ was edited by CRISPR-Cas9, implying that normal $c b s$ expression is required for vasculature development and/or function in A. mexicanus.

Our results suggest that circulatory system dysfunction triggered by cbsa downregulation is important for inhibiting eye growth in Astyanax. We substantiated this conclusion by showing that eye growth is arrested by CRISPR-Cas9 editing of the rap1b gene in SF, which is required for vascular integrity and in mutant form can induce eye hemorrhages during larval development. The reliance of eyes on oxygen, nutrients, or humoral factors provided by blood flow ${ }^{39,40}$ would explain why CF form eyes early in embryogenesis and eye regression begins later during larval development. We also showed that hypoxia gene markers, including the master hypoxia regulator HIF1a, are increased in CF heads or eyes. Therefore, a model is proposed in which deficiencies in oxygen or other factors normally provided by the optic circulatory system are responsible for CF eye regression (Fig. 10). Importantly, we infer that defective circulation, rather than the effects of hemorrhages or aneurysms themselves, is the 


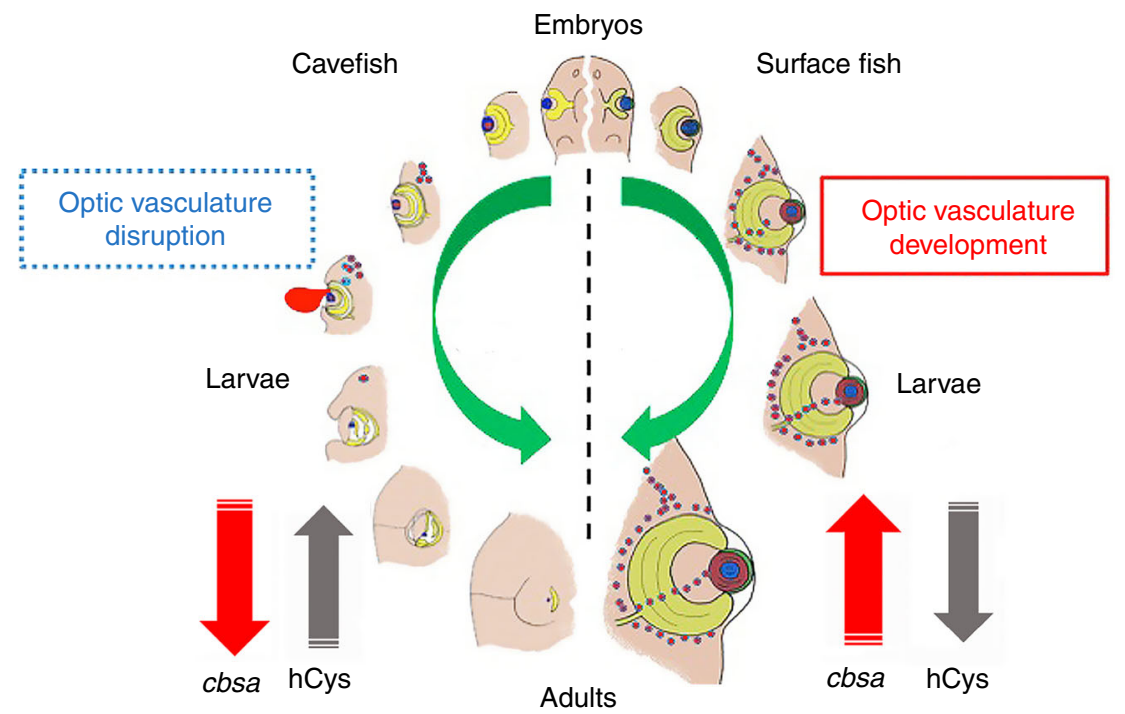

Fig. 10 A model for cavefish eye degeneration. The model illustrates changes in cbsa expression (red upward pointing arrows indicate normal expression in surface fish; red downward pointing arrows indicate downregulated expression in cavefish) resulting in hCys elevation (black downward pointing arrows show normal hCys titers in surface fish; black upward pointing arrows indicate elevated titers in cavefish) and the induction of defects in optic vasculature (red dots surrounding and within developing eyes) that can result in hemorrhages in cavefish (red ellipse; left). Green arrows show trajectories of eye degeneration in cavefish larvae (left) or eye development in surface fish larvae (right).

critical factor induced by the hypomorphic cbsa locus. Interference with eye growth by the induction of localized hypoxic stress may confer an evolutionary benefit for CF by eliminating the high energetic cost of maintaining eyes, as vision is useless in the dark cave environment ${ }^{41,42}$.

Previous studies showed that lens dysfunction is involved in the early stages of CF eye degeneration but also recognized that additional factors must be involved ${ }^{4,10-13}$. Although we have shown here that lens size and apoptosis can be rescued in CF by cbsa overexpression, lens apoptosis was not induced in $c b s$ morphants or cbsa CRISPR-Cas9-edited larvae. Therefore, our results suggest that defects in the CF lens and optic vasculature are governed by different genes and mechanisms that cooperate to produce the complex $\mathrm{CF}$ eye regression trait. It will be interesting to determine whether the other genes contribute to the defective optic vasculature phenotype, the lens dysfunction phenotype, both of these phenotypes, or reveal additional changes involved in vestigial eye formation.

\section{Methods}

Husbandry and general experimental procedures. A. mexicanus SF, PA-CF, TI$\mathrm{CF}, \mathrm{LS}-\mathrm{CF}, \mathrm{JI}-\mathrm{CF}, \mathrm{CH}-\mathrm{CF}$, and MO-CF were obtained from stocks in the Jeffery laboratory. Fish were raised in the laboratory at $25^{\circ} \mathrm{C}$ on a 14 -h light and 10 -h dark photoperiod, and spawning was induced by a temperature increase ${ }^{43}$. Embryos and larvae were raised at $23-25^{\circ} \mathrm{C}$. Some SF embryos were cultured with $400 \mu \mathrm{M}$ phenylthiourea (Sigma-Aldrich, St. Louis, MO) to reduce pigmentation prior to in situ hybridization, blood staining, and immunocytology (see below). Developing SF and PA-CF larvae (40 hpf) were exposed to a $37^{\circ} \mathrm{C}$ heat shock for $1 \mathrm{~h}^{44}$. Animals were maintained and handled according to Institutional Animal Care guidelines of the University of Maryland, College Park (IACUC \#R-NOV-18-59) (Project 1241065-1)

Determination of eye size and morphology. Light microscopy and ImageJ software ${ }^{45}$ was used to measure eye and lens areas (Figs. $6 \mathrm{~m}, \mathrm{n}$ and $7 \mathrm{~d}, \mathrm{~h}$ ) in larvae that were immobilized in 1-2 $\mu \mathrm{g} / \mathrm{ml} \mathrm{MS222}$ (Ethyl 3-aminobenzoate methanesulfonate, Western Chemical Inc., Ferndale, WA) or diameters in larvae that were fixed overnight at $4{ }^{\circ} \mathrm{C}$ in $4 \%$ paraformaldehyde (PFA) following antibody staining (see below). The eye and lens on one side of a head was measured; therefore, the reported $n$ values refer to individual larvae, which are biological replicates. To generate the data in Fig. $6 \mathrm{j}$ and Supplementary Table 2, eye morphologies were determined in MS222-treated specimens by visual inspection or through ImageJ measurements when appropriate. Specimens were grouped into four categories as follows. Category 1: normal eye (eye approximately the same size as controls), category 2: small eye (eye size determined to be less than controls by visual and/or Image J measurements), category 3: category 2-sized eye with lens off center in the retina, and category 4: eye absent. Although categorized on one side of the head, all larvae (except those mentioned as otherwise in the "Results") expressed the reported morphological categories bilaterally.

Separation of larval heads and trunks. SF and PA larvae were dissected into heads and trunks by making a single incision at the base of the cranium (Fig. 3a) with a fine steel scalpel blade while they were immersed in a small volume of fish culture system water containing $1 \mu \mathrm{g} / \mathrm{ml}$ MS222 and viewed under the stereomicroscope. The isolated heads and trunks were immediately immersed in Trizol Reagent (Life Technologies, Grand Island, NY), and RNA was extracted.

cDNA synthesis and RT-PCR. Total RNA was isolated from embryos and larvae using Trizol Reagent, and cDNA was synthesized using the SuperScriptTM III First-Strand Synthesis SuperMix Kit and oligo $(\mathrm{dT})_{20}$ or random hexamer primers (Life Technologies) $^{17}$. Qualitative RT-PCR was done using the PCR Master Kit (Roche Diagnostics GmbH, Mannheim, Germany). The primers used to amplify protein-coding genes from scaffold KB871589.1 in the Ensembl AstMex1.0.2 PACF genome release are listed in Supplementary Table 5. The primers used for PCR amplification of other genes with expression levels determined by qualitative RTPCR are listed in Supplementary Tables 6 and 7. The PCR cycling conditions for qualitative RT-PCR were 1 cycle of initial denaturation at $94^{\circ} \mathrm{C}$ for $2 \mathrm{~min}$, followed by $25-32$ cycles of denaturation $\left(94^{\circ} \mathrm{C}\right.$ for $\left.30 \mathrm{~s}\right)$, annealing $\left(60^{\circ} \mathrm{C}\right.$ for $\left.30 \mathrm{~s}\right)$, and elongation $\left(72^{\circ} \mathrm{C}\right.$ for $\left.30 \mathrm{~s}\right)$, and a final elongation step at $72^{\circ} \mathrm{C}$ for $5 \mathrm{~min}$. The standard was $18 \mathrm{~S}$ rRNA. Quantitative real-time RT-PCR (qPCR) of $c b s a, c b s b$, and hypoxia-sensitive genes was carried using glyceraldehyde 3-phosphate dehydrogenase as a standard ${ }^{15,45}$. The primers used for qPCR are listed in Supplementary Table 7 .

In situ hybridization. RNA probes corresponding to gene-specific sequences in the $c b s a-$, cryaa-, $h s f 2 b p$-, and $c b s b$-coding regions were amplified by PCR from SF cDNA using the primers listed in Supplementary Table 6. The PCR products were cloned into the TOPO vector using the TOPO TA Cloning Kit Dual Promoter (Life Technologies) and confirmed by sequencing. Sense and anti-sense digoxigeninlabeled RNA probes were prepared with SP6 RNA and T7 RNA Polymerases (Roche Diagnostics, Indianapolis, IN). Samples were fixed with $4 \%$ PFA, processed for whole-mount in situ hybridization, and photographed using a light microscope ${ }^{17}$.

F1 hybrid test. F1 hybrid embryos were produced by inseminating SF eggs with PA-CF sperm using in vitro fertilization ${ }^{45}$. RNA was extracted from isolated heads (see above) of SF, PA-CF, and SF X PA-CF F1 hybrids at 60 hpf. Total RNA was extracted, and cDNA was synthesized as described above. The primers for amplification of $c b s a$ by RT-PCR were 5'-CGGATGGTGGAAGATGCAGA-3' (forward) and 5'-CGTAGTGAGCCAGAGGGTTG-3' (reverse). The PCR cycling conditions were 1 cycle of initial denaturation at $94^{\circ} \mathrm{C}$ for $2 \mathrm{~min}$, followed by 28 
cycles of denaturation at $94^{\circ} \mathrm{C}$ for $30 \mathrm{~s}$, annealing at $60^{\circ} \mathrm{C}$ for $30 \mathrm{~s}$, and elongation at $72{ }^{\circ} \mathrm{C}$ for $30 \mathrm{~s}$, and a final elongation step at $72^{\circ} \mathrm{C}$ for $7 \mathrm{~min}$. Amplification of the control 18S rRNA was carried out using $1 \mu \mathrm{l}$ of the synthesized cDNA with primers 5'-GAGTATGGTTGCAAAGCTGAAA-3' (forward) and 5'-CCGGACATCTAA GGGCATCA-3' (reverse). The PCR cycling conditions were 1 cycle of initial denaturation at $94^{\circ} \mathrm{C}$ for $5 \mathrm{~min}$, followed with 25 cycles of denaturation at $94^{\circ} \mathrm{C}$ for $30 \mathrm{~s}$, annealing at $62^{\circ} \mathrm{C}$ for $30 \mathrm{~s}$, and elongation at $72^{\circ} \mathrm{C}$ for $30 \mathrm{~s}$, and a final elongation step at $72^{\circ} \mathrm{C}$ for $7 \mathrm{~min}$.

To determine whether the SF or PA-CF $c b s a$ allele was expressed in hybrids, PCR reactions were performed to amplify a 327-bp region of the cbsa-coding region containing the SF- and $\mathrm{CF}$-specific mononucleotide markers using the forward primer 5'-ACCCTCTGGCTCACTACGAC-3' and the reverse primer 5' TGCGAGCCATAGCAAAGGAC-3' and the Phusion High-Fidelity PCR Master Mix (New England Bio Labs, Ipswich, MA) ${ }^{17}$. The PCR products were purified with the MinElute Gel Extraction Kit (Qiagen, Valencia, CA) and cloned into the Dual Promoter PCR II-TOPO Vector using the TOPO TA Cloning Kit (Life Technologies $)^{17}$. Both PCR products and TOPO plasmids were sequenced. The PCR products were sequenced with forward primer 5'-ACCCTCTGGCTCACTA CGAC-3', and TOPO plasmids were sequenced using the M13-20 and M13rev primers provided with the kit to distinguish which $c b s a$ clones contained the SF or CF marker.

RACE reactions and sequencing. The 5' and 3' ends of SF and PA cbsa mRNA were determined using the SMARTer ${ }^{\mathrm{m}}$ RACE cDNA Amplification Kit (Clontech Laboratories, Inc., Mountain View, CA). Poly A ${ }^{+}$RNA was isolated with the NucleoBond RNA/DNA Kit (Macherey-Nagel, Duren, Germany) and RACE-Ready cDNA was generated using the first-strand cDNA synthesis protocol in the SMAR$\mathrm{Ter}^{\mathrm{rm}}$ RACE cDNA Amplification Kit (Clontech). The primary gene-specific primer for $c b s a$ 5'-RACE was 5'-CGCTCTCTCTGCATCTTCCACCATCCG-3'; and the nested gene-specific primer for $c b s a$ 5'-RACE was 5'-GCTGATTCTGTCCTTCAC ACTGCCGCC-3'; the primary gene specific primer for $c b s a$ 3'-RACE was 5'-TCTC TGCTCCCCTCACTGTTTTGCCCA-3'; and the nested gene-specific primer for 3'RACE was 5'-GGAGACGGATCACTTTGCCCTGGTGGT-3'. The PCR reactions were performed using the Advantage 2 PCR Kit (Clontech). The PCR conditions were 5 cycles at $94^{\circ} \mathrm{C}$ for $30 \mathrm{~s}$ and $72^{\circ} \mathrm{C}$ for $3 \mathrm{~min} ; 5$ cycles at $94^{\circ} \mathrm{C}$ for $30 \mathrm{~s}, 70^{\circ} \mathrm{C}$ for $30 \mathrm{~s}$, and $72{ }^{\circ} \mathrm{C}$ for $3 \mathrm{~min} ; 27$ cycles at $94^{\circ} \mathrm{C}$ for $30 \mathrm{~s}, 68^{\circ} \mathrm{C}$ for $30 \mathrm{~s}$, and $72^{\circ} \mathrm{C}$ for $3 \mathrm{~min}$. For the nested PCR reactions, the cycling conditions were 20 cycles each at $94^{\circ} \mathrm{C}$ for $30 \mathrm{~s}$, $68^{\circ} \mathrm{C}$ for $30 \mathrm{~s}$, and $72^{\circ} \mathrm{C}$ for $3 \mathrm{~min}$. The PCR products were purified with the MinElute PCR Purification Kit (Qiagen) and sequenced with nested gene-specific primers.

Genome walking and sequencing. The SF and PA cbsa gene loci and flanking regions were amplified and sequenced by genome walking ${ }^{17}$. The entire SF and PACF cbsa genomic DNA sequences were amplified using the GenomeWalker ${ }^{\text {Th }}$ Universal Kit (Clontech Laboratories, Mountain View, CA), and the overlapping genomic regions were sequenced step by step using the primers listed in Supplementary Table 8. For the construction of GenomeWalker libraries, genomic DNA was digested with EcoRV, Dra I, PvuII, and StuI I. The GenomeWalker PCR reactions were performed with TaKaRa LA Taq ${ }^{\text {TM }}$ (Takara Bio, Mountain View, CA). The PCR reactions were conducted using the two-step cycle parameters described in the GenomeWalker ${ }^{\mathrm{TM}}$ Kit manual. After obtaining the major bands, the PCR products were inserted into the TOPO vector, and both the PCR products and TOPO plasmids were sequenced.

To amplify the genomic region between $c b s a$ and the adjacent upstream gene gemin8, we designed 14 pairs of primers (Supplementary Table 8) using incomplete sequences available in the Ensembl PA-CF database. The PCR reactions were performed by PCR Master (Roche, Roche Diagnostics GmbH, Mannheim, Germany), and the PCR products were inserted into the TOPO vectors, and both the PCR products and TOPO plasmids were sequenced. After sequencing, lowquality reads and pCRII TOPO vector sequences were removed, the sequences were trimmed, and clean genomic sequence reads were assembled with the DNAstar SeqMan Pro software.

To amplify SF and PA-CF cbsa intron 6, which was not covered by genome walker generated or Ensembl sequences (AstMex1.0.2), we used the forward primer 5'AGATCGTCCGTACCCCTACC-3', which corresponds to a region located in cbsa exon 5, and the reverse primer 5'-TGCCAGCTGAAGTGTGCTTA-3', which corresponds to a region located in intron 11. The PCR reactions were performed using Phusion High-Fidelity PCR Master Mix (New England Biolabs). The PCR cycling conditions were 1 cycle of initial denaturation at $98^{\circ} \mathrm{C}$ for $30 \mathrm{~s}$, followed by 35 cycles each of denaturation at $98^{\circ} \mathrm{C}$ for $8 \mathrm{~s}$, annealing at $68^{\circ} \mathrm{C}$ for $25 \mathrm{~s}$, extension at $72{ }^{\circ} \mathrm{C}$ for $5.5 \mathrm{~min}$, and a final elongation step at $72^{\circ} \mathrm{C}$ for $10 \mathrm{~min}$. The PCR products were purified with the MinElute Gel Extraction Kit (Qiagen) and sequenced.

Indel sequencing in CF populations. Genomic DNA was extracted from tailfin clips of six different CF populations (see above) using the DNeasy Blood \& Tissue Kit (Qiagen). The primers used to amplify the region surrounding the indel in $c b s a$ intron 1 were 5'-GCCTGCATGTGCCAGAGGGG-3' (forward) and 5'-CCGCCG CCAAAACATTGCGT-3' (reverse). The PCR cycling conditions were 1 cycle of initial denaturation at $98^{\circ} \mathrm{C}$ for $30 \mathrm{~s}$, followed by 35 cycles of denaturation $\left(98^{\circ} \mathrm{C}\right.$ for $5 \mathrm{~s}$ ), annealing (at $60^{\circ} \mathrm{C}$ for $15 \mathrm{~s}$ ), extension (at $72^{\circ} \mathrm{C}$ for $15 \mathrm{~s}$ ), and a final extension at $72^{\circ} \mathrm{C}$ for $7 \mathrm{~min}$. The primers used to amplify the region surrounding intron 6 were 5'-CCAGAGGCAGACATGTTTCCGATT-3' (forward) and 5'GGAGGCTGCAGAGTACTGACAGT-3' (reverse), and the PCR cycling conditions were the same as those used for the intron 1 region. The primers used to amplify the region surrounding intron 8 were 5'-TGGCTTCAAGCAAGGGC GGG-3' (forward) and 5'-AGTTGCGGGCAACATCATACCCT-3' (reverse). The PCR cycling conditions were the same as those used to amplify intron 1, except that annealing was done at $58^{\circ} \mathrm{C}$ for $15 \mathrm{~s}$. All PCR reactions were performed using Phusion High-Fidelity PCR Master Mix (New England Biolabs, Ipswich, MA, USA), and the PCR products were purified with the MinElute PCR Purification Kit (Qiagen) and sequenced.

Enhancer identification and transgenesis. Potential enhancers were identified in cbsa non-coding DNA sequence using iEnhancer-2L $\mathrm{L}^{21}$. The predicted enhancer (E in Fig. 3b) in cbsa intron 1, which showed sequence differences between SF and all 6 types of CF, was inserted into the pCMV-GFP vector for expression analysis. The 354-bp region including enhancer E was amplified from SF and PA-CF genomic DNA and inserted into the SpeI site of the pCMV-GFP vector. The pSF-E-CMV CFP and pCF-E-CMV-GFP constructs were confirmed by sequencing. The DNA constructs and transposase mRNA ( $25 \mathrm{ng}$ each) were injected into 1-cell SF embryos, and GFP expression was determined by fluorescence microscopy at 40 hpf. Predicted transcription factor-binding sites in the $c b s a$ intron 1 regions containing indels were identified using the online PROMO (Version 3.0.2) software ${ }^{46,47}$

Gene knockdown and rescue by mRNA injection. Knockdown of the $c b s a$ and $c b s b$ genes was done using the splice inhibiting MOs cbsa MO 5'-CAATGCTAATGCTTTTACCTTCTCC-3', which targeted exon 5 of the $c b s a$ gene, and $c b s b$ MO 5'-AGCCACTGCAAACACACATACATCA-3', which targeted $c b s b$ exon 4. The control MO was 5'-CCTCTTACCTCAGTTACAATTTATA-3'. The MOs were designed and synthesized by Gene Tools, Inc. (Philomath, OR). The $c b s a$ and $c b s b$ MOs were injected into 1-2-cell SF embryos at concentrations of $0.25 \mathrm{mM}$, and the morphants were cultured through the early larval stages.

For RT-PCR validations, the $c b s a$ and $c b s b$ morphants were collected at $40 \mathrm{hpf}$ and total RNA was isolated and cDNA synthesized as described above. The primers used to amplify the region containing the cbsa sequence change were 5'-CGGAT GGTGGAAGATGCAGA-3' (forward) and 5'-CGTAGTGAGCCAGAGGGTTG-3' (reverse). The primers used to amplify the region containing the $c b s b$ sequence change were 5'-GGAAAATTGGAGACACGCCG-3' (forward) and 5'-ATGATGC AGCGGTAACCCTT-3' (reverse).

To prepare $c b s a$ mRNA for rescue experiments, the full-length coding region was amplified from SF $c b s a$ cDNA using 5'-GGGCTCGAGCGAATCAGCACCA CCTGAAC-3' (forward) and 5'-GGGTCTAGAAACCATTCCTTTCAGAGACTG GA-3'(reverse) primers, which included XhoI and XbaI sites. The purified PCR product was digested with $X h o \mathrm{I}$ and $\mathrm{XbaI}$, ligated into the $\mathrm{pCS} 2+$ vector, and the chimeric plasmid was confirmed by sequencing. After linearization with NotI, the capped mRNA was transcribed using the mMESSAGE mMACHINE Sp6 Kit (Life Technologies). After transcription, cbsa mRNA was recovered by $\mathrm{LiCl}$ precipitation, washed in $70 \%$ ethanol, re-suspended in sterile $\mathrm{H}_{2} \mathrm{O}$, and stored at $-20^{\circ} \mathrm{C}$. We injected $c b s a$ mRNA into 1-2-cell $c b s a$ or $c b s b$ morphant SF embryos at $75 \mathrm{ng} / \mu \mathrm{l}$ or into 1 -cell PA-CF embryos at $100 \mathrm{ng} / \mu \mathrm{l}$ in sterile water containing $0.05 \%$ phenol red ${ }^{17}$.

CRISPR-Cas9 gene editing. Mutations in the SF $c b s a$ and rap $1 b$ genes were induced by CRISPR-Cas9 editing using two guide RNAs (single guide RNA (sgRNA)) to obtain maximal efficiency ${ }^{48}$. The cbsa sgRNAs were designed using ENSEMBL ENSAMXT00000008310: the sequence of the first cbsa sgRNA was "AAGUGCGAGUACUUCAACGC," and the sequence of the second $c b s a$ sgRNA was "UGCGAGUACUUCAACGCCGG." The $r a p 1 b$ sgRNAs were designed using ENSEMBL ENSAMXT00000030949.1: the sequence of the first rap1b sgRNA was "GGAACAAUUCACAGCCAUGA," and the sequence of the second rap1b sgRNA was "AGGAACAAUUCACAGCCAUG." We co-injected 50 pg of each sgRNA and 300 pg of Cas9 protein (Cas9 nuclease 2NLS, S. pyrogenes, Synthego Corp., Redwood City, CA) into one-cell-stage SF embryos. CRISPR-Cas9-injected and control un-injected embryos from the same clutch were cultured at $25^{\circ} \mathrm{C}$, phenotyped at $3.5 \mathrm{dpf}$ by microscopy, genomic DNA was extracted from injected and control larvae, and nested PCR was used to amplify the edited sites. For $c b s a$-edited larvae, the flanking primers were 5'-GTATGCAGACAGCACTACAGG-3' (forward) and 5'-ACCTGGACGACTGGAATGTTA-3' (reverse), and the nested primers were 5'ATGCAGACAGCACTACAGGC-3' (forward) and 5'-TCTTCTCAAACCCCTCG TCAC-3' (reverse). For rap1b-edited larvae, the flanking primers were 5'-GCACC CCAAAGGCTTGTTTA-3' (forward) and 5'-GCTACCTACAAAGGAGGCAGA3' (reverse), and the nested primers were 5'-ACCCCTGTCATTATGTGGGC-3' (forward) and 5'-ATGAATGGATGAGTGGCGAGG-3' (reverse).

hCys determination and injection. hCys was quantified in SF, SF cbs morphants, PA-CF, and TI-CF larvae using the Homocysteine ELISA Kit (Cell Biolabs Inc., San 
Diego, CA). Samples containing 50 larvae were homogenized in phosphatebuffered saline (PBS), the homogenates were centrifuged at $10,000 \times g$ for $15 \mathrm{~min}$ at $4{ }^{\circ} \mathrm{C}$, and the supernatant fraction was collected and assayed immediately according to the instructions of the manufacturer.

L-homocysteine and L-cysteine (both purchased from Sigma Aldrich) were diluted to $1-5 \mathrm{mM}$ in sterile water containing $0.05 \%$ phenol red, microinjected into 1-2-cell SF embryos at a final concentration of $5 \mathrm{mM}$, and the effects on eye development were determined in SF larvae at $4 \mathrm{dpf}$.

Microangiography. Microangiography ${ }^{49}$ was performed on SF, PA-CF, or $c b s$ morphants mounted in $1.2 \%$ low-melt agarose prepared in zebrafish embryo medium ${ }^{50}$. Embryos were injected with Qtracker 655 or 705 vascular labels (ThermoFisher Scientific) in the dorsal aorta around the yolk extension area using a pressure injector (World Precision Instruments, Friedberg, Germany). Injected embryos were immediately observed using an upright Leica TCS SP5 confocal microscope. Vascular leakages were analyzed in and around the head regions. At least three to five injected embryos were examined per dose in each experiment.

Macrophage and red blood cell staining. Vital staining for macrophages was done by incubating 4-10 dpf PA-CF larvae with $25 \mu \mathrm{g} / \mathrm{ml}$ Neutral Red in fish system tank water for $12 \mathrm{~h}$ at room temperature in the dark ${ }^{51}$. Embryos were stained for red blood cells for $15 \mathrm{~min}$ with $0.6 \mathrm{mg} / \mathrm{ml}$ o-dianisidine (Sigma, St. Louis, MO, USA), $0.01 \mathrm{M}$ sodium acetate ( $\mathrm{pH} 4.5), 0.65 \%$ hydrogen peroxide, and $40 \%(\mathrm{v} / \mathrm{v})$ ethanol in the dark ${ }^{52}$. After staining, the embryos were rinsed in PBS, post-fixed in $4 \%$ PFA for $1 \mathrm{~h}$, rinsed in PBS, and stored in $80 \%$ glycerol.

Antibody staining. SF and PA-CF larvae from 1.5 to $12 \mathrm{dpf}$ were fixed in $4 \%$ PFA overnight at $4{ }^{\circ} \mathrm{C}$, dehydrated through an increasing series of methanol concentrations to $100 \%$ methanol, and stored at $-20^{\circ} \mathrm{C}$. The larvae were rehydrated, blocked with superblock solution containing $5 \%$ goat serum, and incubated in a 1:100 dilution of rabbit polyclonal anti-HIFla primary antibody (Catalogue Number 114977, Abcam, Cambridge, UK) for $48 \mathrm{~h}$ at $4{ }^{\circ} \mathrm{C}$, rinsed with superblock, then incubated with 1:200 dilution of Alexa Fluor 488 goat anti-rabbit IgG secondary antibody (ThermoFisher, Waltham, MA) overnight at $4{ }^{\circ} \mathrm{C}$, rinsed with PBS, $0.1 \%$ Triton $\mathrm{X}-100,0.2 \%$ bovine serum albumin, and visualized by fluorescence microscopy.

Lens deletion and apoptosis detection. The lens was removed from the optic cup on one side of SF embryos at about $30 \mathrm{hpf}$ by microdissection using Tungsten needles ${ }^{53}$, and hemorrhaging was assayed by light microscopy from 2 to $8 \mathrm{dpf}$.

Lens apoptosis was determined by vital staining of SF, PA-CF, and $c b s$ morphants at $40 \mathrm{hpf}$ with $5 \mu \mathrm{g} / \mathrm{ml}$ Lysotracker Red DND 99 (Invitrogen, Carlsberg, CA) for $30 \mathrm{~min}$ in the dark ${ }^{45}$. Stained larvae were anesthetized in MS222 (see above) and mounted on glass slides for imaging.

Reporting summary. Further information on research design is available in the Nature Research Reporting Summary linked to this article.

\section{Data availability}

The authors declare that all data supporting the findings of this study are available within the article and its Supplementary Information files or from the corresponding author upon reasonable request. Data are reported in figures, Supplementary Figures, and Supplementary Tables, and raw sequence data have been deposited in the NCBI database under accession codes: MK801789 (SF $c b s a$ mRNA), MK801790 (PA-CF $c b s a$ mRNA), MN186089 (SF cbsa genomic region), and MN186090 (PA-CF cbsa genomic region). The source data for Figs. 1, 2, 3c-e, 4b-h, 5, 6k-r, 7, 8, 9, and Supplementary Fig. 4a, b, g, h are provided as a source data file.

Received: 1 October 2019; Accepted: 4 May 2020;

Published online: 02 June 2020

\section{References}

1. Krishnan, J. \& Rohner, N. Cavefish and the basis for eye loss. Philos. Trans. R. Soc. Lond. B Biol. Sci. 372, 20150487 (2017).

2. Jeffery, W. R. Regressive evolution in Astyanax cavefish. Annu. Rev. Genet. 43, 25-47 (2009).

3. Yamamoto, Y. \& Jeffery, W. R. Central role for the lens in cave fish eye degeneration. Science 289, 631-633 (2000).

4. Strickler, A. G., Yamamoto, Y. \& Jeffery, W. R. The lens controls cell survival in the retina: evidence from the blind cavefish Astyanax. Dev. Biol. 311, 512-523 (2007).

5. Alunni, A. et al. Developmental mechanisms for retinal degeneration in the blind cavefish Astyanax mexicanus. J. Comp. Neurol. 505, 221-233 (2007).
6. Gross, J. B. The complex origin of Astyanax cavefish. BMC Evol. Biol. 12, 105 (2012).

7. Herman, A. et al. The role of gene flow in rapid and repeated evolution of cave-related traits in Mexican tetra, Astyanax mexicanus. Mol. Ecol. 27, 4397-4416 (2018).

8. Porter, M. L. \& Crandall, K. A. Lost along the way: the significance of evolution in reverse. Trends Ecol. Evol. 18, 541-547 (2003).

9. Kim, E. B., et al. Genome sequencing reveals insights into physiology and longevity of the naked mole rat. Nature 479: 223-227 (2011).

10. Borowsky, R. \& Wilkens, H. Mapping a cave fish genome: polygenic systems and regressive evolution. J. Hered. 93, 19-21 (2002).

11. Protas, M., Conrad, M., Gross, J. B., Tabin, C. \& Borowsky, R. Regressive evolution in the Mexican cave tetra, Astyanax mexicanus. Curr. Biol. 17, 452-454 (2007).

12. O’Quin, K. E., Yoshizawa, M., Doshi, P. \& Jeffery, W. R. Quantitative genetic analysis of retinal degeneration in the blind cavefish Astyanax mexicanus. PLoS ONE 8, e57281 (2013).

13. Borowsky, R. Restoring sight in blind cavefish. Curr. Biol. 18, R23-R24 (2008)

14. Gore, A. V. et al. An epigenetic mechanism for cavefish eye degeneration. Nat. Ecol. Evol. 2, 1155-1160 (2018).

15. McGaugh, S. E. et al. The cavefish genome reveals candidate genes for eye loss. Nat. Commun. 5, 5307 (2014).

16. Hallmann, K. et al. A homozygous splice-site mutation in CARS2 is associated with progressive myoclonic epilepsy. Neurology 83, 2183-2187 (2014).

17. Ma, L., Parkhurst, A. \& Jeffery, W. R. The role of a lens survival pathway including sox 2 and $\alpha A$-crystallin in the evolution of cavefish eye degeneration. EvoDevo 5, 28 (2014).

18. Yoshima, T., Yura, T. \& Yanagi, H. Novel testis-specific protein that interacts with heat shock factor 2. Gene 214, 139-146 (1998).

19. Prabhudesai, $\mathrm{S}$. et al. Cystathionine $\beta$-synthase is necessary for axis development in vivo. Front. Cell Dev. Biol. 6, 14 (2018).

20. Wittkopp, P. J., Haerum, B. K. \& Clark, A. G. Evolutionary changes in cis and trans gene regulation. Nature 430, 85-88 (2004).

21. Liu, B., Fang, L., Long, R., Lan, X. \& Chou, K. C. iEnhancer-2L: a two-layer predictor for identifying enhancers and their strength by pseudo k-tuple nucleotide composition. Bioinformatics 32, 362-369 (2016).

22. Mudd, S. H., Levy, H. L. \& Kraus, J. P. in The Metabolic and Molecular Bases of Inherited Disease (eds Scriver, C. R. et al.) 2007-2056 (McGraw-Hill, New York, 2001).

23. Mudd, S. H. et al. The natural history of homocystinuria due to cystathionine $\beta$-synthase deficiency. Am. J. Hum. Genet. 37, 1-31 (1985).

24. Kraus, J. P. \& Kožich, V. in Homocysteine in Health and Disease (eds R. Carmel, R. \& Jacobsen, D. W.) 223-243 (Cambridge University Press, Cambridge, 2001).

25. Cai, Y. et al. Homocysteine-responsive ATF3 gene expression in human vascular endothelial cells: activation of c-Jun $\mathrm{NH}_{2}$-terminal kinase and promoter response element. Blood 96, 2140-2148 (2000).

26. Kamath, A. F. et al. Elevated levels of homocysteine compromise blood-brain barrier integrity in mice. Blood 107, 591-593 (2006).

27. Tawfik, A. et al. Alterations of retinal vasculature in cystathionine-betasynthase mutant mice, a model of hyperhomocysteinemia. Inves. Ophthalmol. Vis. Sci. 54, 939-949 (2013).

28. Gore, A. V., Lampugnani, M. G., Dye, L., Dejana, E. \& Weinstein, B. M. Combinatorial interaction between CCM pathway genes precipitates hemorrhagic stroke. Dis. Model. Mech. 1, 275-281 (2008).

29. Long, Y. et al. Transcriptional events co-regulated by hypoxia and cold stresses in zebrafish larvae. BMC Genomics 16, 385 (2015).

30. Ton, C., Stamatiou, D. \& Liew, C. C. Gene expression profile of zebrafish exposed to hypoxia during development. Physiol. Genomics 13 , 97-106 (2003).

31. Alvarez-Tejado, M. et al. Hypoxia induces the activation of the phosphatidylinositol 3-kinase/Akt cell survival pathway in PC12 cells: protective role in apoptosis. J. Biol. Chem. 276, 22368-22374 (2001).

32. Deuel, J. W. et al. Different target specificities of haptoglobin and hemopexin define a sequential protection system against vascular hemoglobin toxicity. Free Radic. Biol. Med. 89, 931-943 (2015).

33. Wittenberg, B. A. \& Wittenberg, J. B. Transport of oxygen in muscle. Ann. Rev. Physiol. 51, 857-878 (1989).

34. Mazurais, D. et al. Identification of hypoxia-regulated genes in the liver of common sole (Solea solea) fed different dietary lipid contents. Mar. Biotechnol. (NY) 16, 277-288 (2014).

35. Watanabe, M. et al. Mice deficient in cystathionine $\beta$-synthase: animal models for mild and severe homocyst(e)inemia. Proc. Natl Acad. Sci. USA 92, 1585-1589 (1995).

36. Enokido, Y. et al. Cystathionine B-synthase, a key enzyme for homocysteine metabolism, is preferentially expressed in the radial glia/astrocyte lineage of 
the developing mouse CNS. FASEB J. https://doi.org/10.1096/fj.05-3724fje. (2016)

37. Schmatolla, E. Dependence of tectal neural differentiation on optic innervation in teleost fish. J. Embryol. Exp. Morphol. 27, 555-576 (1972).

38. Soares, D., Yamamoto, Y., Strickler, A. G. \& Jeffery, W. R. The lens has a specific influence on optic nerve and tectum development in the blind cavefish Astyanax. Dev. Neurosci. 26, 308-317 (2004).

39. Luo, X., Shen, Y. M., Jiang, M. N., Lou, X. F. \& Shen, Y. Ocular blood flow autoregulation mechanisms and methods. J. Ophthalmol. 2015, 864871 (2015).

40. Saint-Genez, M. \& D'Amore, P. A. Development and pathology of hylaloid, choroidal and retinal vasculature. Int. J. Dev. Biol. 48, 1045-1058 (2004).

41. Yu, D. Y. \& Cringle, S. J. Oxygen distribution and consumption within the retina in vascularised and avascular retinas and in animal models of retinal disease. Prog. Retin. Eye Res. 20, 175-208 (2001).

42. Moran, D., Softley, R. \& Warrant, E. J. The energetic cost of vision and the evolution of eyeless Mexican cavefish. Sci. Adv. 1, e1500363 (2015).

43. Jeffery, W. R., Strickler, A. G., Guiney, S., Heyser, D. G. \& Tomarev, S. I. Prox 1 in eye degeneration and sensory organ compensation during development and evolution of the cavefish Astyanax. Dev. Genes Evol. 210, 223-230 (2000).

44. Hooven, T. A., Yamamoto, Y. \& Jeffery, W. R. Blind cavefish and heat shock protein chaperones: a novel role for hsp90alpha in lens apoptosis. Int. J. Dev. Biol. 48, 731-738 (2004).

45. Ma, L. et al. Maternal genetic effects in Astyanax cavefish development. Dev. Biol. 441, 209-220 (2018).

46. Messeguer, X. et al. PROMO: detection of known transcription regulatory elements using species-tailored searches. Bioinformatics 18, 333-334 (2002).

47. Farré, D. et al. Identification of patterns in biological sequences at the ALGGEN server: PROMO and MALGEN. Nucleic Acids Res. 31, 3651-3653 (2003).

48. Varshney, G. K. et al. A high-throughput functional genomics workflow based on CRISPR/Cas9-mediated targeted mutagenesis in zebrafish. Nat. Protoc. 11, 2357-2375 (2016).

49. Jung, H. M. et al. Imaging blood vessels and lymphatic vessels in the zebrafish. Methods Cell Biol. 133, 69-103 (2016).

50. Westerfield, M. The Zebrafish Book: A Guide for the Laboratory Use of Zebrafish (Danio rerio) 5 edn (University of Oregon Press, Eugene, OR, 2007).

51. Herbomel, P., Thisse, B. \& Thisse, C. Zebrafish early macrophages colonize cephalic mesenchyme and developing brain, retina, and epidermis through a M-CSF receptor-dependent invasive process. Dev. Biol. 238, 274-288 (2001).

52. O’Brien, B. R. A. Identification of haemoglobin by its catalase reaction with peroxide and o-dianisidine. Stain Technol. 36, 57-61 (1961).

53. Yamamoto, Y. \& Jeffery, W. R. Probing teleost eye development by lens transplantation. Methods 28, 420-426 (2002).

\section{Acknowledgements}

We are grateful to Ruby Dessiatoun for fish maintenance and husbandry; to Annie Hasselbalch and Sara Soueidan for technical support; and to Eric Haag, Scott Juntti, and Xiao Liu for comments on a draft manuscript. This work was supported by an NIH grant EY024941 to W.R.J.

\section{Author contributions}

L.M., W.R.J., A.V.G., and B.M.W. conceived the project and L.M., A.V.G., W.R.J., D.C., J.S., M.N., and C.M.v.d.W. performed the research. W.R.J. and L.M. wrote the manuscript with input from all authors.

\section{Competing interests}

The authors declare no competing interests.

\section{Additional information}

Supplementary information is available for this paper at https://doi.org/10.1038/s41467 020-16497-x.

Correspondence and requests for materials should be addressed to W.R.J.

Peer review information Nature Communications thanks Florencia Cavodessi and the other anonymous reviewer(s) for their contribution to the peer review of this work.

Reprints and permission information is available at http://www.nature.com/reprints

Publisher's note Springer Nature remains neutral with regard to jurisdictional claims in published maps and institutional affiliations.

Open Access This article is licensed under a Creative Commons Attribution 4.0 International License, which permits use, sharing, adaptation, distribution and reproduction in any medium or format, as long as you give appropriate credit to the original author(s) and the source, provide a link to the Creative Commons license, and indicate if changes were made. The images or other third party material in this article are included in the article's Creative Commons license, unless indicated otherwise in a credit line to the material. If material is not included in the article's Creative Commons license and your intended use is not permitted by statutory regulation or exceeds the permitted use, you will need to obtain permission directly from the copyright holder. To view a copy of this license, visit http://creativecommons.org/ licenses/by/4.0/.

(C) The Author(s) 2020 